AperTO - Archivio Istituzionale Open Access dell'Università di Torino

\title{
Ab Initio Calculation of the Ultraviolet-Visible (UV-vis) Absorption Spectrum, Electron-Loss Function, and Reflectivity of Solids
}

\section{This is the author's manuscript}

Original Citation:

Availability:

This version is available http://hdl.handle.net/2318/1573381

since 2016-06-28T10:39:58Z

Published version:

DOI:10.1021/acs.jctc.5b00199

Terms of use:

Open Access

Anyone can freely access the full text of works made available as "Open Access". Works made available under a Creative Commons license can be used according to the terms and conditions of said license. Use of all other works requires consent of the right holder (author or publisher) if not exempted from copyright protection by the applicable law. 
This is the author's final version of the contribution published as:

Ferrari, Anna Maria; Orlando, Roberto; Rérat, Michel. Ab Initio Calculation of the Ultraviolet-Visible (UV-vis) Absorption Spectrum, Electron-Loss Function, and Reflectivity of Solids. JOURNAL OF CHEMICAL THEORY AND COMPUTATION. 11 (7) pp: 3245-3258.

DOI: $10.1021 /$ acs.jctc.5b00199

The publisher's version is available at:

http://pubs.acs.org/doi/abs/10.1021/acs.jctc.5b00199

When citing, please refer to the published version.

Link to this full text:

http://hdl.handle.net/2318/1573381 


\title{
Ab initio calculation of the UV-visible absorption
}

\section{spectrum, electron-loss function and reflectivity of}

\section{solids}

\author{
Anna Maria Ferrari, ${ }_{i}^{\dagger \dagger}$ Roberto Orlando, ${ }^{\dagger}$ and Michel Rérat ${ }^{\ddagger}$ \\ Dipartimento di Chimica IFM, Università di Torino and NIS -Nanostructured Interfaces and \\ Surfaces - Centre of Excellence, http://www.nis.unito.it Via P. Giuria 7, 10125 Torino, Italy, and \\ Equipe de Chimie Physique, IPREM UMR5254, Université de Pau et des Pays de l'Adour, 64000 \\ Pau, France \\ E-mail:
}

${ }^{a}$ Corresponding author: anna.ferrari@unito.it

\footnotetext{
${ }^{*}$ To whom correspondence should be addressed

†Dipartimento di Chimica IFM, Università di Torino and NIS -Nanostructured Interfaces and Surfaces - Centre of Excellence, http://www.nis.unito.it Via P. Giuria 7, 10125 Torino, Italy

־Equipe de Chimie Physique, IPREM UMR5254, Université de Pau et des Pays de l’Adour, 64000 Pau, France
} 
May 20, 2015

\begin{abstract}
The field frequency has recently been taken into account in the coupled-perturbed HartreeFock or Kohn-Sham method implemented in the CRYSTAL code ${ }^{1}$ for calculating the highfrequency dielectric constant of semi-conductors up to the first electronic transitions. In this work, we document how the code has been generalized and improved in order to compute the full UV-visible absorption spectrum, the electron loss function and the reflectivity from the real and imaginary parts of the electric response property. We show how spectra are modified when the crystalline orbital relaxation due to the dynamic electric field is taken into account, and how this modification increases with the percentage of Hartree-Fock exchange in the unperturbed hybrid hamiltonian.
\end{abstract}

Keywords: Ab initio calculation, coupled-perturbed Hartree-Fock and Kohn-Sham method (CPHF/CPKS), time-dependent density functional theory (TDDFT), excitons, hybrid hamiltonian, CRYSTAL code, UV-visible absorption spectrum, electron loss function $(E L F)$, reflectivity, $\mathrm{Si}, \mathrm{SiC}$ and LiF.

\title{
Introduction
}

The coupled-perturbed Hartree-Fock (CPHF) method $^{2}$ at first order of perturbation has been implemented a few years ago in the CRYSTAL $\operatorname{code}^{3}$ for calculating the electronic contribution to the so-called high-frequency polarizability (or $\varepsilon^{\infty}$ dielectric constant) of closed and open shell periodic systems. The CRYSTAL ${ }^{1,4}$ code, which uses atomic orbitals (AO) for building Bloch functions (BF), solves the Hartree-Fock (HF) and Kohn-Sham (KS) equations, the latter with different kinds of hybrid hamiltonians in the framework of the density functional theory (DFT). The coupled-perturbed method has, then, been adapted at the first and second orders of perturbation for the KS equation. ${ }^{5}$ More recently, the $\omega$-frequency of the field has been introduced in the $\mathrm{CPHF}(\mathrm{KS})$ method so allowing the study of the real part of $\varepsilon^{\infty}(\omega)$, or of the refractive index $n=\sqrt{\varepsilon^{\infty}}$, as a 
function of the light wavelength. ${ }^{6}$ Applications to the photoelastic tensor and its dependence on frequency is described in Refs. 7,8.

Near resonances the dielectric response, related to second-order transition amplitudes due to a time-dependent electric field perturbation, is a complex function depending on a damping factor related to the finite life time of excited states, which depends on several physical interactions in solids (phonon coupling, defects, ...), not easy to evaluate. The imaginary part of $\varepsilon^{\infty}(\omega)$ leads to the UV-visible spectrum, i.e. to the oscillator strengths versus transition or pole energies. In the following section, we show how it is computed in the full range of UV-visible energies for periodic systems, by slightly modifying the frequency-dependent $\mathrm{CPHF}(\mathrm{KS})$ method already implemented in the CRYSTAL code. The performance of the method, similar to the time-dependent Hartree-Fock (TDHF) method $^{9}$ or to the configuration interaction singles (CIS) method ${ }^{10,11}$ as implemented in a molecular context, or to the time-dependent DFT (TDDFT) and equivalent Bethe-Salpeter equation (BSE), ${ }^{12,13}$ as used for periodic compounds, is discussed in the present work by considering bulk Silicon (Si), SiC and LiF. The effect of the computational parameters controlling the calculation will be considered in the Si case for which high quality experimental results ${ }^{14-16}$ and many previous computations ${ }^{17}$ are available. We will discuss, in particular, the influence of the percentage of "exact" exchange in the hybrid functionals on the UV-visible absorption spectrum (UV), electron loss function $(E L F)$ and reflectivity $(R)$, starting from the observation of Marques et al. ${ }^{18}$ and the very recent work of Skone et al. ${ }^{19}$ It is worth noting that, although the necessity of using hybrid functionals to compute reasonable band gaps has been frequently underlined recently, most of the applications compute the dynamic polarizability up to the first resonance only. ${ }^{20-25}$ A systematic investigation of the effect of the "exact" exchange on the UV, ELF and $R$ functions of solids (from small gap semiconductors to insulators) is still lacking.

In "Frequency dependent CPHF(KS)" section, we recall first the frequency-dependent CPHF(KS) basic equations implemented in the CRYSTAL code and how the exciton effects can appear in UV. Then, we discuss the effect of the computational parameters in the case of Si. And in the last section, $\mathrm{UV}, E L F$ and $R$ for a small gap semiconductor $(\mathrm{Si})$, a wide gap semiconductor $(\mathrm{SiC})$ and 
an insulator (LiF) are compared.

\section{Frequency dependent CPHF(KS)}

\section{The optical dielectric constant}

The cartesian components of the static polarizability $\alpha_{u v}$, and the related elements of the dielectric constant, are calculated as follows in the $\mathrm{AO}$ basis set $(\mu, v)$ :

$$
\begin{gathered}
\alpha_{u v}=-\frac{4}{n_{k}} \sum_{\mathbf{k}}^{B Z} \mathscr{R}_{e}\left\{\sum_{\mu v} \sum_{i}^{\mathrm{occ}} \sum_{a}^{\mathrm{virt}} C_{\mu i}^{\mathbf{k} *} \Omega_{\mu \nu}^{\mathbf{k}, v} C_{v a}^{\mathbf{k}} U_{i a, \mathbf{k}}^{u}\right\} \\
\varepsilon_{u v}^{\infty}=\delta_{u v}+\frac{4 \pi}{V} \alpha_{u v}
\end{gathered}
$$

where $n_{k}$ is the number of $\mathbf{k}$ points in the first Brillouin Zone (BZ) and the indices $i(j)$ and $a(b)$ span the occupied and virtual crystalline orbitals, respectively, and where $V$ is the volume of the cell containing twice more electrons than occupied orbitals per $k$-point for closed-shell systems. $U_{\mathbf{k}}^{u}$ is the unknown anti-hermitian off-diagonal-block matrix that linearly transforms the unperturbed eigenvectors $C^{\mathbf{k}}$ under the effect of the electric field perturbation represented by matrix $\Omega^{\mathbf{k}}$ (and later defined in the text):

$$
\left.\mathbb{C}_{\mu i}^{\mathbf{k}, u} \equiv \frac{\partial C_{\mu i}^{\mathbf{k}}}{\partial \varepsilon_{u}}\right|_{0}=\sum_{l}^{\text {all }} C_{\mu l}^{\mathbf{k}} U_{i l, \mathbf{k}}^{u}
$$

The off-diagonal (occupied-virtual) blocks $U_{i a, \mathbf{k}}^{u}$, defined as

$$
U_{i a, \mathbf{k}}^{u}=\frac{\sum_{\mu \nu} C_{\mu a}^{\mathbf{k} * \mathbb{F}_{\mu \nu}^{\mathbf{k}, u} C_{v i}^{\mathbf{k}}}}{\varepsilon_{i_{\mathbf{k}}}^{(0)}-\varepsilon_{a_{\mathbf{k}}}^{(0)}}
$$


are functions of both the energy gap $\left(\varepsilon_{i_{\mathbf{k}}}^{(0)}-\varepsilon_{a_{\mathbf{k}}}^{(0)}\right)$, and the perturbed Fock matrix (Hartree-Fock hamiltonian), ${ }^{3}$

$$
\begin{gathered}
\left.\mathbb{F}_{\mu \nu}^{\mathbf{k}, u} \equiv \frac{\partial F_{\mu \nu}^{\mathbf{k}}}{\partial \varepsilon_{u}}\right|_{0}=\Omega_{\mu \nu}^{\mathbf{k}, u}+ \\
\sum_{\mathbf{g}} e^{\imath \mathbf{k} \cdot \mathbf{g}} \sum_{\lambda \rho} \sum_{\mathbf{k}^{\prime}}^{B Z} \sum_{\mathbf{g}^{\prime}} e^{-\imath \mathbf{k}^{\prime} \cdot \mathbf{g}^{\prime}} \sum_{j}\left(\mathbb{C}_{\lambda j}^{\mathbf{k}^{\prime}, u} C_{\rho j}^{\mathbf{k}^{\prime} *}+C_{\lambda j}^{\mathbf{k}^{\prime}} \mathbb{C}_{\rho j}^{\mathbf{k}^{\prime} u, *}\right) \sum_{\mathbf{g}^{\prime \prime}}\left[2\left(\mu^{\mathbf{0}} v^{\mathbf{g}} \mid \lambda^{\mathbf{g}^{\prime \prime}} \rho^{\mathbf{g}^{\prime}+\mathbf{g}^{\prime \prime}}\right)-\left(\mu^{\mathbf{0}} \lambda^{\mathbf{g}^{\prime \prime}} \mid v^{\mathbf{g}} \rho^{\mathbf{g}^{\prime}+\mathbf{g}^{\prime \prime}}\right)\right]
\end{gathered}
$$

Moving from the Bloch (AO) basis to the crystalline orbitals (CO) basis, we obtain:

$$
\mathbb{F}_{i a, \mathbf{k}}^{u}=\sum_{\mu \nu} C_{\mu a}^{\mathbf{k} *} \mathbb{F}_{\mu \nu}^{\mathbf{k}, u} C_{v i}^{\mathbf{k}}=\Omega_{i a, \mathbf{k}}^{u}+\sum_{\mathbf{k}^{\prime}}^{B Z} \sum_{j} \sum_{b} U_{j b, \mathbf{k}^{\prime}}^{u} B_{i a, \mathbf{k}}^{j b, \mathbf{k}^{\prime}}+U_{j b, \mathbf{k}^{\prime}}^{u *} B_{i a, \mathbf{k}}^{b j, \mathbf{k}^{\prime}}
$$

where $\Omega_{i a, \mathbf{k}}^{u}=\left\langle i_{\mathbf{k}}\left|u+\imath \frac{d}{d \mathbf{k}_{u}}\right| a_{\mathbf{k}}\right\rangle$ is the transition moment operator for periodic systems ${ }^{26}(u=x, y$ or $z$ ) between occupied $i_{\mathbf{k}}$ and virtual $a_{\mathbf{k}}$ crystalline orbitals with unperturbed eigenvalues $\varepsilon_{i_{\mathbf{k}}}^{(0)}$ and $\varepsilon_{a_{\mathbf{k}}}^{(0)}$, respectively, for each $\mathbf{k}$-point of the periodic system. This expression of the $\Omega$-operator holds if the wave vector $\mathbf{q}$ of the field is close to zero, ${ }^{27} \mathrm{i}$. e. the wavelength is much larger than the unit cell size, condition that is valid for the static case and for the UV-visible light. The $B_{i a, \mathbf{k}}^{j b, \mathbf{k}^{\prime}}$ and $B_{i a, \mathbf{k}}^{b j, \mathbf{k}^{\prime}}$ terms are defined as follows:

$$
\begin{aligned}
& B_{i a, \mathbf{k}}^{j b, \mathbf{k}^{\prime}}=\left[2\left\langle i_{\mathbf{k}} b_{\mathbf{k}^{\prime}} \mid a_{\mathbf{k}} j_{\mathbf{k}^{\prime}}\right\rangle-\left\langle i_{\mathbf{k}} b_{\mathbf{k}^{\prime}} \mid j_{\mathbf{k}^{\prime}} a_{\mathbf{k}}\right\rangle\right] \\
& B_{i a, \mathbf{k}}^{b j, \mathbf{k}^{\prime}}=\left[2\left\langle i_{\mathbf{k}} j_{\mathbf{k}^{\prime}} \mid a_{\mathbf{k}} b_{\mathbf{k}^{\prime}}\right\rangle-\left\langle i_{\mathbf{k}} j_{\mathbf{k}^{\prime}} \mid b_{\mathbf{k}^{\prime}} a_{\mathbf{k}}\right\rangle\right]
\end{aligned}
$$

The notation: $\langle i j \mid a b\rangle$ is used for the bielectronic integral $\left\langle i(1) j(2)\left|\frac{1}{r_{12}}\right| a(1) b(2)\right\rangle$.

Then, $U_{\mathbf{k}}^{u}$ (Eq. 4) for a given k-point depends on $\mathbb{F}_{\mathbf{k}}^{u}$ (Eq. 6), which depends in turn on the $U_{\mathbf{k}^{\prime}}^{u}$ matrices for all k-points. Hence, a Self-Consistent Coupled-Perturbed (SC-CP) process is to be carried out (CPHF or CPKS). At the first cycle of the process, the derivative of the density matrix 
with respect to the field is equal to zero and there is no bielectronic contribution in $\mathbb{F}_{i a, \mathbf{k}}^{u}$. This step of unrelaxed orbitals corresponds to the Sum Over States approximation (SOS).

If the field is frequency $(\omega)$-dependent, the time-dependent perturbation theory leads to the two following $U^{ \pm}$matrices (see Refs. 9,28 for the molecular or $\mathbf{k}=\mathbf{0}$ cases, and Ref. 6 for periodic systems):

$$
U_{i a, \mathbf{k}}^{u \pm[n]}=\lim _{\eta \rightarrow 0^{+}} \frac{\Omega_{i a, \mathbf{k}}^{u}+\sum_{\mathbf{k}^{\prime}} \sum_{j}^{o c c} \sum_{b}^{v i r t}\left(U_{j b, \mathbf{k}^{\prime}}^{u \pm[n-1]} B_{i a, \mathbf{k}}^{j b, \mathbf{k}^{\prime}}+U_{j b, \mathbf{k}^{\prime}}^{u \mp *[n-1]} B_{i a, \mathbf{k}}^{b j, \mathbf{k}^{\prime}}\right)}{\varepsilon_{i_{\mathbf{k}}}^{(0)}-\varepsilon_{a_{\mathbf{k}}}^{(0)} \pm \omega+\imath \eta}
$$

where the number $\eta$ is a damping factor producing the peak broadening when the inverse of life time of excited states is taken into account. This is not easily evaluated in solids and usually the energy resolution of the experimental equipment is used instead ( $\eta \simeq 0.1 \mathrm{eV}$ in $\mathrm{UV}$-visible). The equivalent of Eq. 2 in the frequency-dependent case is:

$$
\mathscr{R} e\left(\varepsilon_{u v}^{\infty}(\omega)\right)=\delta_{u v}+\frac{4 \pi}{V} \alpha_{u v}(\omega)=\delta_{u v}-\frac{8 \pi}{V} \frac{1}{n_{k}} \mathscr{R}_{e} \sum_{\mathbf{k}}^{B Z} \sum_{i}^{\text {occ }} \sum_{a}^{\text {virt }} \Omega_{a i, \mathbf{k}}^{v}\left(U_{i a, \mathbf{k}}^{u+}+U_{i a, \mathbf{k}}^{u-}\right)
$$

Note that the dielectric matrix elements are real if $\eta=0: \varepsilon_{u v}^{\infty}(\omega)=\mathscr{R} e\left(\varepsilon_{u v}^{\infty}(\omega)\right)$.

To better understand the origin of the energy of the pole, let us separate the "diagonal" $(i=j, a=b$, $\mathbf{k}=\mathbf{k}^{\prime}$ ) bielectronic term from all others in Eq. 9 at convergence of the iterative SC-CP process (where: $U_{i a, \mathbf{k}}^{u \pm[n]} \simeq U_{i a, \mathbf{k}}^{u \pm[n-1]} \simeq U_{i a, \mathbf{k}}^{u \pm}$ ):

$$
U_{i a, \mathbf{k}}^{u \pm}=\frac{\Omega_{i a, \mathbf{k}}^{u}+U_{i a, \mathbf{k}}^{u \pm} B_{i a, \mathbf{k}}^{i a, \mathbf{k}}+U_{i a, \mathbf{k}}^{u \mp *} B_{i a, \mathbf{k}}^{a i, \mathbf{k}}+\sum_{\left(\mathbf{k}^{\prime}, j, b\right) \neq(\mathbf{k}, i, a)}\left(U_{j b, \mathbf{k}^{\prime}}^{u \pm} B_{i a, \mathbf{k}}^{j b, \mathbf{k}^{\prime}}+U_{j b, \mathbf{k}^{\prime}}^{u \mp *} B_{i a, \mathbf{k}}^{b j, \mathbf{k}^{\prime}}\right)}{\varepsilon_{i_{\mathbf{k}}}^{(0)}-\varepsilon_{a_{\mathbf{k}}}^{(0)} \pm \omega+\imath \eta}
$$

This matrix element can be written as follows ${ }^{1}$ :

$$
U_{i a, \mathbf{k}}^{u \pm}=\frac{\Omega_{i a, \mathbf{k}}^{u}+U_{i a, \mathbf{k}}^{u \mp *} B_{i a, \mathbf{k}}^{a i, \mathbf{k}}+\sum_{\left(\mathbf{k}^{\prime}, j, b\right) \neq(\mathbf{k}, i, a)}\left(U_{j b, \mathbf{k}^{\prime}}^{u \pm} B_{i a, \mathbf{k}}^{j b, \mathbf{k}^{\prime}}+U_{j b, \mathbf{k}^{\prime}}^{u \mp *} B_{i a, \mathbf{k}}^{b j, \mathbf{k}^{\prime}}\right)}{\varepsilon_{i_{\mathbf{k}}}^{(0)}-\varepsilon_{a_{\mathbf{k}}}^{(0)}-B_{i a, \mathbf{k}}^{i a, \mathbf{k}} \pm \omega+\imath \eta}
$$

\footnotetext{
${ }^{1}$ In going from Eq. 11 to Eq. 12 , we use: $x=\frac{a+b x}{c} \Rightarrow x(1-b / c)=\frac{a}{c}$ which leads to $x=\frac{a}{c-b}$
} 


\section{Resonance}

Before looking at the expression of $\mathscr{I} m\left(\varepsilon_{u v}^{\infty}(\omega)\right)$ that provides the UV spectrum, we want to underline the relevance of Eq. 12. Although Eqs. 11 and 12 are formally equivalent, Eq. 12 makes the SC-CP procedure much clearer. In the SOS approximation for real $U_{i a, \mathbf{k}}^{u+}(\eta \rightarrow 0)$ :

$$
U_{i a, \mathbf{k}}^{u+}=\frac{\Omega_{i a, \mathbf{k}}^{u}}{\varepsilon_{i_{\mathbf{k}}}^{(0)}-\varepsilon_{a_{\mathbf{k}}}^{(0)}+\omega}
$$

the resonance occurs at $\omega=\varepsilon_{a_{\mathbf{k}}}^{(0)}-\varepsilon_{i_{\mathbf{k}}}^{(0)}$. However, Eq. 12 shows that, when the system responds to the perturbation (the SC-CP scheme is active), the resonance is shifted by $B_{i a, \mathbf{k}}^{i a, \mathbf{k}}$. The $U_{i a, \mathbf{k}}^{u+}$ element becomes infinite near the new resonance $\omega=\omega_{i a, \mathbf{k}}=\varepsilon_{a_{\mathbf{k}}}^{(0)}-\varepsilon_{i_{\mathbf{k}}}^{(0)}+B_{i a, \mathbf{k}}^{i a, \mathbf{k}}$ where the denominator tends to zero. We can do the further step by considering now the behaviour of $U_{i a, \mathbf{k}}^{u-}$ in Eq. 12. At resonance, the denominator is not null (due to a negative sign of $\omega$ ), but the numerator tends to infinity due to the presence of $U_{i a, \mathbf{k}}^{u+}$. So we have: $U_{i a, \mathbf{k}}^{u-} \simeq U_{i a, \mathbf{k}}^{u+*} B_{i a, \mathbf{k}}^{a i, \mathbf{k}} /\left(\varepsilon_{i_{\mathbf{k}}}^{(0)}-\varepsilon_{a_{\mathbf{k}}}{ }^{(0)}-B_{i a, \mathbf{k}}^{i a, \mathbf{k}}-\omega_{i a, \mathbf{k}}\right)$. The $U_{i a, \mathbf{k}}^{u-*}$ element which appears in the numerator of Eq. 12 for $U_{i a, \mathbf{k}}^{u+}$ is equal to $-U_{i a, \mathbf{k}}^{u+} B_{i a, \mathbf{k}}^{a i, \mathbf{k}} / 2 \omega_{i a, \mathbf{k}}$. Then, the expression of $U_{i a, \mathbf{k}}^{u+}$ in Eq. 12 becomes (see footnote 1):

$$
U_{i a, \mathbf{k}}^{u+} \simeq \frac{\Omega_{i a, \mathbf{k}}^{u}}{\varepsilon_{i_{\mathbf{k}}}^{(0)}-\varepsilon_{a_{\mathbf{k}}}^{(0)}-B_{i a, \mathbf{k}}^{i a, \mathbf{k}}+\left(B_{i a, \mathbf{k}}^{a i, \mathbf{k}}\right)^{2} / 2 \omega_{i a, \mathbf{k}}+\omega}
$$

This means that the pole is shifted with respect to SOS by $B_{i a, \mathbf{k}}^{i a, \mathbf{k}}=2\left\langle i_{\mathbf{k}} a_{\mathbf{k}} \mid a_{\mathbf{k}} i_{\mathbf{k}}\right\rangle-\left\langle i_{\mathbf{k}} a_{\mathbf{k}} \mid i_{\mathbf{k}} a_{\mathbf{k}}\right\rangle$ (first order correction), and by something depending on $B_{i a, \mathbf{k}}^{a i, \mathbf{k}}=\left\langle i_{\mathbf{k}} i_{\mathbf{k}} \mid a_{\mathbf{k}} a_{\mathbf{k}}\right\rangle$ (see Eqs. 7 and 8; note also the interchange between $i a$ and $a i)$.

In solids, where the density of states (in the valence and conduction bands) is a continuous function of the energy, the summation on $\mathbf{k}^{\prime}, b$ and $j$ cannot be disregarded because several other transitions $j_{\mathbf{k}^{\prime}} \rightarrow b_{\mathbf{k}^{\prime}}$ may occur around $\omega_{i a, \mathbf{k}}$ for which we can assume that: $U_{j b, \mathbf{k}^{\prime}}^{u+} / U_{i a, \mathbf{k}}^{u+} \simeq \Omega_{j b, \mathbf{k}^{\prime}}^{u} / \Omega_{i a, \mathbf{k}}^{u}$ (in Eq. 14, the denominator for $U_{i a, \mathbf{k}}^{u+}$ and $U_{j b, \mathbf{k}^{\prime}}^{u+}$ is roughly the same). The resulting bielectronic terms $U_{j b, \mathbf{k}^{\prime}}^{u+} B_{i a, \mathbf{k}}^{j b, \mathbf{k}^{\prime}}$ in the numerator of $U_{i a, \mathbf{k}}^{u+}$ (Eq. 12) can be moved outside the summation once weighted by the density $\rho\left(\omega_{i a, \mathbf{k}}\right)$, representing the number of transitions $j_{\mathbf{k}^{\prime}} \rightarrow b_{\mathbf{k}^{\prime}}$ for which the transition 
energy $\omega_{j b, \mathbf{k}^{\prime}}$ is close to $\omega_{i a, \mathbf{k}}$. Then, at resonance Eq. 11 can be replaced by (for $\eta=0$ ):

$$
U_{i a, \mathbf{k}}^{u+}=\frac{\bar{\Omega}_{i a, \mathbf{k}}^{u}}{\varepsilon_{i_{\mathbf{k}}}^{(0)}-\varepsilon_{a_{\mathbf{k}}}^{(0)}-\rho\left(\omega_{i a, \mathbf{k}}\right) \bar{B}_{i a, \mathbf{k}}^{j b, \mathbf{k}^{\prime}}+\omega}
$$

In the above equation $\bar{B}_{i a, \mathbf{k}}^{j b, \mathbf{k}^{\prime}}$ includes all the contributions from bielectronic integral between orbitals involved in transitions with an energy value close to the pole:

$$
\omega_{i a, \mathbf{k}}=\varepsilon_{a_{\mathbf{k}}}^{(0)}-\varepsilon_{i_{\mathbf{k}}}^{(0)}+\rho\left(\omega_{i a, \mathbf{k}}\right) \bar{B}_{i a, \mathbf{k}}^{j b, \mathbf{k}^{\prime}}
$$

and $\bar{\Omega}_{i a, \mathbf{k}}^{u}$ is equal to $\Omega_{i a, \mathbf{k}}^{u}$ corrected by all the other bielectronic $B_{i a, \mathbf{k}}^{j^{\prime} b^{\prime}, \mathbf{k}^{\prime}}$ and $B_{i a, \mathbf{k}}^{b^{\prime} j^{\prime}, \mathbf{k}^{\prime}}$ contributions corresponding to transitions $j_{\mathbf{k}^{\prime}} \rightarrow b_{\mathbf{k}^{\prime}}$ far in energy from $\omega_{i a, \mathbf{k}}$.

\section{Imaginary part of the dielectric constant}

When $\eta \neq 0$, near resonance the imaginary part of the complex dielectric constant can be written as follows (see Eqs. 10 and 15):

$$
\mathscr{I}_{m}\left(\varepsilon_{u v}^{\infty}(\omega)\right)=\frac{8 \pi}{V n_{k}} \sum_{\mathbf{k}}^{B Z} \sum_{i}^{\text {occ virt }} \sum_{a}^{\eta} \frac{\mathscr{R}_{e}\left(\Omega_{i a, \mathbf{k}}^{v *} \bar{\Omega}_{i a, \mathbf{k}}^{u}\right)}{\left(\omega_{i a, \mathbf{k}}-\omega\right)^{2}+\eta^{2}}
$$

The non-resonant $U_{i a, \mathbf{k}}^{u-}$ terms have been neglected in the previous equation since they are expected to give negligible contributions to the resonance. Actually they can be calculated directly without any approximation from the product of $\Omega_{i a, \mathbf{k}}^{u}$ by $U_{i a, \mathbf{k}}^{u-}$ in Eq. 11 .

The case of the resonant $U_{i a, \mathbf{k}}^{u+}$ term is more delicate. Expression 11 seems to show that the damping factor becomes important when $\omega_{i a, \mathbf{k}}=\varepsilon_{a_{\mathbf{k}}}^{(0)}-\varepsilon_{i_{\mathbf{k}}}^{(0)}$, Eq. 12, however, shows that a shift by $\rho\left(\omega_{i a, \mathbf{k}}\right) \bar{B}_{i a, \mathbf{k}}^{j b, \mathbf{k}^{\prime}}$ must be taken into account. If, then, Eq. 11 is used as such in the calculation, it turns out that the imaginary part of $U_{i a, \mathbf{k}}^{u+}$ is changing sign in the $\left[\varepsilon_{a_{\mathbf{k}}}^{(0)}-\varepsilon_{i_{\mathbf{k}}}^{(0)}+\rho\left(\omega_{i a, \mathbf{k}}\right) \bar{B}_{i a, \mathbf{k}}^{j b, \mathbf{k}^{\prime}}, \varepsilon_{a_{\mathbf{k}}}^{(0)}-\right.$ $\left.\varepsilon_{i_{\mathbf{k}}}^{(0)}\right]$ interval (remember that $\mathscr{I}_{m}\left(\varepsilon_{u v}^{\infty}(\omega)\right)$ must be a positive quantity). An alternative equivalent formulation permits to avoid large positive to negative oscillations around the resonance. From Eq. 
15, we have:

$$
\left|\omega_{i a, \mathbf{k}}-\omega\right|=\left|\bar{\Omega}_{i a, \mathbf{k}}^{u} / U_{i a, \mathbf{k}}^{u+}(\eta=0)\right|
$$

The denominator in Eq. 17 can be recast as:

$$
\mathscr{I}_{m}\left(\varepsilon_{u v}^{\infty}(\omega)\right)=\frac{8 \pi}{V n_{k}} \sum_{\mathbf{k}}^{B Z} \sum_{i}^{\text {occ virt }} \sum_{a} \frac{\eta \mathscr{R}_{e}\left(\Omega_{i a, \mathbf{k}}^{v *} \bar{\Omega}_{i a, \mathbf{k}}^{u}\right)}{\left|\bar{\Omega}_{i a, \mathbf{k}}^{u} / U_{i a, \mathbf{k}}^{u+}(\eta=0)\right|^{2}+\eta^{2}}=\frac{8 \pi}{V n_{k}} \sum_{\mathbf{k}}^{B Z} \sum_{i}^{\text {occ virt }} \sum_{a} \frac{\eta \mathscr{R}_{e}\left(\Omega_{i a, \mathbf{k}}^{v *} \bar{\Omega}_{i a, \mathbf{k}}^{u}\right)}{\bar{\Omega}_{i a, \mathbf{k}}^{u *} \bar{\Omega}_{i a, \mathbf{k}}^{u}}\left|U_{i a, \mathbf{k}}^{u+}\right|^{2}
$$

where in the second step we used the square modulus of Eq. 15 (but with $\eta \neq 0$ ). The above function is proportional to $\eta$ and, then, close to zero when far from resonance $\left(\left|\bar{\Omega}_{i a, \mathbf{k}}^{u} / U_{i a, \mathbf{k}}^{u+}\right| \gg \eta\right)$; near the resonance, it is inversely proportional to $\eta$ and large. In that case, the imaginary part of the dielectric constant is proportional to the product of the transition moments $\Omega_{i a, \mathbf{k}}^{u *} \bar{\Omega}_{i a, \mathbf{k}}^{u}$ multiplied by the new density of transitions $\rho\left(\omega_{i a, \mathbf{k}}\right)$ (as the $i_{\mathbf{k}}$ and $a_{\mathbf{k}}$ functions extend to all the cases such as: $\left.\omega_{i a, \mathbf{k}}=\varepsilon_{a_{\mathbf{k}}}^{(0)}-\varepsilon_{i_{\mathbf{k}}}^{(0)}+\rho\left(\omega_{i a, \mathbf{k}}\right) \bar{B}_{i a, \mathbf{k}}^{j b, \mathbf{k}^{\prime}}\right)$. Till now, no approximation has been introduced in the expression of the imaginary part of the dielectric constant, but the presence of $\bar{\Omega}_{i a, \mathbf{k}}$ shows that we need to calculate the correction on the transition moment $\Omega_{i a, \mathbf{k}}^{u}$ due to bielectronic integral contributions $\left(U_{j b, \mathbf{k}^{\prime}}^{u \pm} B_{i a, \mathbf{k}}^{j b, \mathbf{k}^{\prime}}\right.$ and $\left.U_{j b, \mathbf{k}^{\prime}}^{u \pm} B_{i a, \mathbf{k}}^{b j, \mathbf{k}^{\prime}}\right)$ involving non-resonant $j_{\mathbf{k}^{\prime}} \rightarrow b_{\mathbf{k}^{\prime}}$ transitions (see Eq. 12). This correction should be small if these non-resonant transitions are far from the present $i_{\mathbf{k}} \rightarrow a_{\mathbf{k}}$ resonant transition and not too numerous (the case of molecules but probably not of periodic systems). Nevertheless, they have been neglected in the imaginary part of the dielectric constant of which the expression (diagonal component) finally becomes:

$$
\mathscr{I}_{m}\left(\varepsilon_{u u}^{\infty}(\omega)\right) \simeq \frac{8 \pi}{V n_{k}} \sum_{\mathbf{k}}^{B Z} \sum_{i}^{\text {occ virt }} \sum_{a}^{\text {ing }} \eta\left|U_{i a, \mathbf{k}}^{u+}\right|^{2}
$$

when replacing $\bar{\Omega}_{i a, \mathbf{k}}^{u}$ with $\Omega_{i a, \mathbf{k}}^{u}$ in Eq. 19.

A decrease in the numerator of $\mathscr{I}_{m}\left(\varepsilon_{u u}^{\infty}(\omega)\right)$ due to coupling corrections in Eq. 19 on the transition moment should lead, however, to the decrease of the peak height and to the increase of its full width 
at half maximum (FWHM), and vice versa, with no drastic change of the integral of the spectrum.

\section{Oscillator strength}

The multiplication of the imaginary part of the dielectric constant (Eq. 20) by $2 \omega /(4 \pi / V)$ leads to the oscillator strength at each $\omega$-frequency, i.e. to the UV spectrum. The integration of the spectrum over $\omega$ leads to:

$$
\frac{1}{\pi} \int_{0}^{\infty} 2 \omega \mathscr{I}_{m}\left(\varepsilon_{u u}^{\infty}(\omega)\right) /(4 \pi / V) d \omega=\frac{2}{n_{k}} \sum_{\mathbf{k}}^{B Z} \sum_{i}^{\text {occ }} \sum_{a}^{\operatorname{virt}} \frac{1}{\pi} \int_{0}^{\infty} \frac{2 \omega \eta\left|\Omega_{a i, \mathbf{k}}^{u}\right|^{2}}{\left(\omega_{i a, \mathbf{k}}-\omega\right)^{2}+\eta^{2}} d \omega=\frac{2}{n_{k}} \sum_{\mathbf{k}}^{B Z} \sum_{i}^{\text {occ virt }} \sum_{a} 2 \omega_{i a, \mathbf{k}}\left|\Omega_{a i, \mathbf{k}}^{u}\right|^{2}
$$

each transition intensity being a Lorentzian function with height $1 / \eta$ and width $2 \eta$ for which the integration is $\pi$. In the case of pure DFT, orbitals are obeying the hypervirial relation (for a large enough $\mathrm{AO}$ basis $\operatorname{set}^{27}$ ):

$$
\left(\varepsilon_{i_{\mathbf{k}}}^{(0)}-\varepsilon_{a_{\mathbf{k}}}^{(0)}\right) \Omega_{i a, \mathbf{k}}^{u}=-\nabla_{i a, \mathbf{k}}^{u}
$$

where $\nabla^{u}=d / d u$ acts in the direct space.

Then, the sum over oscillator strengths obtained in Eq. 21 is equal to the number of valence electrons involved in UV (Thomas-Reiche-Kuhn sum rule):

$$
\frac{2}{n_{k}} \sum_{\mathbf{k}}^{B Z} \sum_{i}^{\text {occ virt }} \sum_{a}^{2} 2\left(\varepsilon_{a_{\mathbf{k}}}^{(0)}-\varepsilon_{i_{\mathbf{k}}}^{(0)}\right)\left|\Omega_{i a, \mathbf{k}}^{u}\right|^{2}=\frac{2}{n_{k}} \sum_{\mathbf{k}}^{B Z} \sum_{i}^{\text {occ }} \sum_{a}^{\text {virt }} 2 \nabla_{i a, \mathbf{k}}^{u} \Omega_{a i, \mathbf{k}}^{u}=\frac{2}{n_{k}} \sum_{\mathbf{k}}^{B Z} \sum_{i}^{\text {occ }} 2\left\langle i_{\mathbf{k}}\left|\nabla^{u} \Omega^{u}\right| i_{\mathbf{k}}\right\rangle=n_{e}
$$

if the basis set is complete. The number of electrons $n_{e}$ on the right-hand side of the above equation is obtained considering that ${ }_{l} \nabla^{u}=-\imath d / d u$ and $\Omega^{u}=u+\imath d / d k_{u}\left(=\imath e^{\imath \mathbf{k} \cdot \mathbf{r}} \nabla_{k_{u}} e^{-l \mathbf{k} \cdot \mathbf{r}}\right)$ are hermitian operators. With hybrid hamiltonians, the UV surface is not equal to the number of electrons because Eq. 22 is not valid due to the non-commutativity of r-position and HF-exchange (non local) operators, and also because $\bar{\Omega}_{i a, \mathbf{k}}^{u}$ has been replaced by $\Omega_{i a, \mathbf{k}}^{u}$ in Eq. 19. It follows an amplification 
of the peak intensities (with respect to SOS ones obtained with the same $\eta$ value), which can be renormalized a posteriori by the value of the full UV integration.

\section{Computational details}

Calculations were performed with a development version of the periodic ab initio CRYSTAL14 code. Several functionals have been tested: the local LDA, ${ }^{29,30}$ the gradient corrected $\mathrm{PBE}^{31}$ and the hybrid B3LYP. ${ }^{32,33}$

In addition, the dependence of the dielectric function on the amount of exact exchange incorporated into the hybrid functional has been tested by considering a different percentage, $x$, of exact exchange with respect to the standard value (25\% in B3LYP): $x=10$ (F10LYP), $x=15$ (F15LYP), $x=40$ (F40LYP) and $x=50$ (F50LYP).

The following basis sets have been used (see Refs. 27,34): 8-8411-11 [1s4sp2d] (Si), 6-21-11 [1s2sp2d] (C), 7-311-1 [1s2sp1d] (F) and a 6-1-1 [1s1sp1p] (Li).

In the CRYSTAL code the level of accuracy in evaluating the Coulomb and exchange integrals is controlled by five $T$ parameters $(T 1=T 2=T 3, T 4, T 5=2 T 4)$ (see Ref. 1). The first two parameters control the Coulomb series and the latter three the exchange one. Several selections of $T=(T 1, T 4)$ have been tested in the present calculations ranging from $T=(8,8)$ to $T=(10,25)$ (used as a reference).

The k-grid mesh is controlled in the CRYSTAL code by the shrinking factor IS. Increasing values of $I S$ have been considered: 16, 36 and 100, corresponding to 145, 1240 and $22000 \mathrm{k}$ points for a cubic lattice, respectively.

A damping factor $\eta$ (Eq. 9) selected in the range of $0.03-0.1 \mathrm{eV}$ has been found to provide stable results $(\eta=0.055 \mathrm{eV}$ is used if not otherwise specified).

The difficulty to obtain the limit of $U_{i a, \mathbf{k}}^{u+}$ iteratively appears when $\omega=\varepsilon_{a_{\mathbf{k}}}^{(0)}-\varepsilon_{i_{\mathbf{k}}}^{(0)}$, i.e. when the numerator and denominator in Eq. 9 are both equal to zero (if $\eta$ is null), while this limit is well defined and equal to $-\Omega_{i a, \mathbf{k}}^{u} /\left(\rho\left(\omega_{i a, \mathbf{k}}\right) \bar{B}_{i a, \mathbf{k}}^{j b, \mathbf{k}^{\prime}}\right)$ near this transition energy (use Eq. 15 with 
$\left.\omega=\varepsilon_{a_{\mathbf{k}}}^{(0)}-\varepsilon_{i_{\mathbf{k}}}^{(0)}\right)$. Actually, the SC-CP process is similar to the numerical Gauss-Seidel method ${ }^{35}$ for solving large linear equations systems: $A X=B$, where $X$ represents the unknown vector (the $U$-vector in the present work), $B$ the dynamic field perturbation, and $A$ the projected timedependent hamiltonian $H-i \hbar d / d t$ in the single occupied-virtual excitation basis set. Gauss-Seidel is particularly efficient when the matrix $A$ is diagonal dominant. However, if one or more diagonal terms of the $A$ matrix is close to zero, the solution does not converge any more. This happens near resonances and a mathematical algorithm is required to force the convergence of the iterative process. The epsilon-algorithm- $2^{36,37}$ using the X-vector obtained from the previous 2-iterations has been used after each four SC-CP iterations in the whole range of the UV-visible absorption energy, and convergence has been reached for most of the field frequencies. The SC-CP process prevents, then, from the storage of a huge hamiltonian matrix (with off-diagonal $B_{i a, \mathbf{k}}^{j b, \mathbf{k}^{\prime}}$ elements) before its diagonalization.

In order to facilitate comparison between UV spectra, the imaginary part of the dielectric constant is first broadened with lorentzian functions. Then, the integration of $2 \omega \mathscr{I}_{m}(\varepsilon(\omega))$ over $\omega$ (which theoretically leads to the number of valence electrons involved in the spectrum; see Eqs. 21-23) allows to renormalize each plot of $\mathscr{I}_{m}(\varepsilon)$ a posteriori.

\section{Applications}

\section{Silicon}

\section{Electron struture}

The band structure (BS) and projected density of states of Silicon (PDOS) are reported in Figure S1 of ESI. Detail of BS close to the Fermi level $E_{f}$ have been sketched considering several special points in the reciprocal space, namely: $\Gamma=\left(\begin{array}{lll}0 & 0 & 0\end{array}\right), X=(0,1 / 2,1 / 2), W=(1 / 4,3 / 4,1 / 2), U=(1 / 4$, $5 / 8,5 / 8)$ and $K=(3 / 8,3 / 4,3 / 8)$. Here we summarize the main features. The valence bands VB (bands from 11 to 14 ) and the conduction bands CB are largely due to 3 sp orbitals. VBs cover a 
range of about $13 \mathrm{eV}$ and $\mathrm{CB}$ extends for more than $25 \mathrm{eV}$ (B3LYP, see Figure S1 and Table S1 of ESI). The 2 sp orbitals contribute to core states (about $100 \mathrm{eV}$ below $E_{f}$ ) and to virtual states (50-100 eV above $E_{f}$ ). The highest occupied VB and the lowest unoccupied CB will be hereafter referred to as HOMO and LUMO for analogy with the molecular case. Si has an indirect gap $E_{g}(\Gamma \rightarrow W)=2.02 \mathrm{eV}$ and a direct band gap in $\Gamma, E_{g}(\Gamma)=3.77 \mathrm{eV}$; at $L, E_{g}(L)$ is equal to $3.96 \mathrm{eV}$ and, then, quite close to $E_{g}(\Gamma)$. At the other special k points, the gap is much larger: $E_{g}(X)=5.07$ $\mathrm{eV}$ and $E_{g}(U(X))=5.10 \mathrm{eV}$; the $E_{H O M O \rightarrow L U M O+1}(L)=6.18 \mathrm{eV}$ is the other lowest energy difference between VB and CB bands (B3LYP results, see Table S1 of ESI). PBE results are qualitatively similar to B3LYP but energy differences are much smaller (by about $1.5 \mathrm{eV}$ ). Intermediate values are provided by F10LYP and F15LYP functionals: for instance, F15LYP provides $E_{g}(\Gamma)=3.52 \mathrm{eV}$, $E_{g}(X)=5.07 \mathrm{eV}, E_{g}(U(X))=5.10 \mathrm{eV}$ and $E_{H O M O \rightarrow L U M O+1}(L)=5.86 \mathrm{eV}$, Table $\mathrm{S} 1$ of ESI.

\section{UV spectrum}

In this section we discuss the computed UV spectrum $(U V)$ and the influence of the computational parameters.

- Sampling the reciprocal space.

$\mathscr{I}_{m}\left(\varepsilon^{\infty}\right)$-CP computed with the F10LYP functional and with several choices of $I S(16,36$ and 100 ), is reported in Figure 1. With $I S=16$, the computed spectrum is significantly different with respect to the reference one $(I S=100)$, due to the presence of spurious peaks, that disappear by moving to $I S=36$ : in this case both positions and heights of all the peaks are correctly predicted.

- Truncation of the Coulomb and exchange series.

Figure 1 reports $\mathscr{I}_{m}\left(\varepsilon^{\infty}\right)(\mathrm{CP}-\mathrm{F} 10 \mathrm{LYP})$ as a function of the integral tolerance parameters $T$. The effect of the Coulomb series is a minor one as shown by comparing $T=(7,25)$ and $T=(10,25)$. When the exchange integrals are involved in going from $T=(8,8)$ to $T=(8,45)$, the 
main effect is an enhancement of the intensity of the two main peaks, and more generally of the surface of the whole $U V$ spectrum as it is explained in subsection "Oscillator strengths". However, the same ratio $(0.20 \pm 0.01)$ between the main peaks is roughly preserved when $T$ increases.

- The damping factor $\eta$.

The combination of $\eta$ and number of points used to sample the energy range determines the resolution of $U V$. In Figure $1, \mathscr{I}_{m}(\varepsilon)$-CP is reported for several values of $\eta$, namely $\eta=0.055$, 0.082 and $0.11 \mathrm{eV}$. For F10LYP, no significant change of the spectrum was found, while the F15LYP-spectrum acquires more detail when $\eta$ is smaller: each peak can be decomposed in one or more components. However, the position of the main peaks, and their relative intensity are again not affected by this choice.

- The DFT functionals.

$\mathscr{I}_{m}\left(\varepsilon^{\infty}\right)$-SOS and $\mathscr{I}_{m}\left(\varepsilon^{\infty}\right)$-CP spectra, computed with different functionals (LDA, PBE, F10LYP, F15LYP and B3LYP) have been reported in Figure $2 . \mathscr{I}_{m}\left(\varepsilon^{\infty}\right)$-SOS spectra show nearly the same shape for all functionals. With respect to B3LYP, LDA and PBE are rigidly shifted to lower energies and F10LYP (F15LYP) to intermediate ones: the $\mathscr{I}_{m}\left(\varepsilon^{\infty}\right)$-SOS spectrum resembles the band structure as provided by the selected functional, and is described by the vertical unperturbed $i_{\mathbf{k}} \rightarrow a_{\mathbf{k}}$ monoexcitations, the transition moment of which is $\Omega_{i a, \mathbf{k}}^{u}$. Considering now the SC-CP calculation of $\mathscr{I}_{m}\left(\varepsilon^{\infty}\right)$, we see that when pure DFT functionals are employed, $U V$ does not show any significant differences with respect to the SOS counterpart (see Fig. 2). The $\mathscr{I}_{m}\left(\varepsilon^{\infty}\right)$-CP spectrum of hybrid functionals, on the contrary, takes a new shape due to the presence of new peaks and enhancement of the previous SOS-intensities, particularly evident in the 3.4-5.0 eV region.

F15LYP $\mathscr{I}_{m}\left(\varepsilon^{\infty}\right)$-CP computed with increasing resolutions are reported in Figures 1 and 2 . The highly resolved spectrum ( $I S=36$, damping factor $\eta=0.5 \mathrm{mHa}$ ) shows several peaks: a high intense doublet at 3.69 and $3.75 \mathrm{eV}$, another intense doublet at 4.55 and $4.62 \mathrm{eV}$, a peak at $4.93 \mathrm{eV}$ and a 
low intense one at $5.76 \mathrm{eV}$.

Comparison of the positions of each peak with the silicon BS (Figure S1 and Table S1 of ESI) allows the following assignments. The peaks in the first intense doublet are assigned to $H O M O \rightarrow L U M O$ transitions from oscillators in a narrow region around $\Gamma$ the former, and to $L$ the latter, whereas the ones in the second doublet derive from $H O M O \rightarrow L U M O$ transitions in a large region around $X$; the 5.76 peak corresponds to $H O M O \rightarrow L U M O+1$ transitions in a narrow region close to $L$. In Ref. 16 a detailed analysis of the experimental spectrum is reported. The spectrum is composed by several doublets: at 3.40 and $3.45 \mathrm{eV}$, at 4.18 and $4.22 \mathrm{eV}$ and at 4.44 and $4.60 \mathrm{eV}$, and a peak at $5.4 \mathrm{eV}$. Computed peaks are then in a very good agreement with the observed ones despite a slight shift (0.3-0.4 eV) towards higher energies. The F10LYP spectrum, see Figure 2, shows poles at $3.45,4.49$ and $5.48 \mathrm{eV}$. Although their positions are closer to the experimental ones, the intensity of the first peak is too low compared to those at higher energy. The surface of the spectrum (in a polarized direction) has been found to be larger than the number of valence electrons by $10 \%$ with SOS, and in order to make the comparison with the experimental spectra easier (see Refs. 14,16), the computed $\mathrm{CPHF}(\mathrm{KS})$-spectrum has been normalized a posteriori to match Eq. 23 (see Fig. 3). The shape of the F15LYP spectrum, even if slightly shifted to higher energies, reproduces the observed one quite nicely, confirming that the approximation made on the imaginary part of the dielectric constant (Eq. 20) is satisfactory with this percentage of exchange (15\%), whereas it is less satisfactory with F10LYP.

Also the region below $E_{g}$ has been analyzed. Much higher resolution is required to check for the presence of bound excitons below $E_{g}$. F15LYP highly resolved spectrum $(I S=150, \eta=0.01 \mathrm{mHa})$ computed in a narrow region close to the band gap edge shows a first resonance downshifted with respect to $E_{g}=3.5234 \mathrm{eV}$ by $0.5 \mathrm{meV}$. Thus, the optical spectrum below $E_{g}$ shows the presence of a very weakly bound exciton. 


\section{Electron energy loss function, $E L F(\omega)$ and reflectivity $R(\omega)$ spectrum}

$E L F(\omega)$ is the $\mathscr{I}_{m}\left(-1 / \varepsilon^{\infty}(\omega)\right)$ function. It has a peak at $\mathscr{R}_{e}\left(\varepsilon^{\infty}\right)=0$; the corresponding frequency is the plasmon pole $\omega_{p}$. It describes the collective harmonic oscillations of electrons with respect to the fixed lattice of ions. Above the plasmon frequency, the external field oscillates too fast for the electrons to follow and a material loses its reflectivity $R(\omega)=\left|\left(1-\sqrt{\varepsilon^{\infty}(\omega)}\right) /\left(1+\sqrt{\varepsilon^{\infty}(\omega)}\right)\right|^{2}$ dramatically. The calculated ELF corresponds to the intensity in the electron energy loss spectroscopy (low valence energy) for which the wavelength of the field is still very large compared to the size of a unit cell (wavelength vector modulus $q \simeq 0$ ). As for $U V$ we analyze now the effect of the computational set up on the computed $E L F(\omega)$ and $R(\omega)$ spectra.

- Computational parameters

$E L F(\omega)$ and $R(\omega)$ spectra computed with several selection of $T$ and $I S$ parameters have been reported in Figure 4. $E L F(\omega)$ and $R(\omega)$ spectra computed with several selection of $T$ and IS parameters have been reported in Figure 4. The effect of integral tolerance parameters controlling the exchange series has been analyzed by considering increasing values of $T$, $T=(8,15),(8,25)$ and $(8,35)$ (the truncation of the Coulomb series has been shown to affect the spectra is a minor way, Figure 1). The shape of both $E L F(\omega)$ and $R(\omega)$ spectra in the whole $0-25 \mathrm{eV}$ energy range, is marginally affected by the selection of $T$; variations of the absolute intensities are particularly visible in the low frequency region, which however, turn out to be negligible when considering the $T=(8,25)$ and $T=(8,35)$ spectra.

The $E L F$ seems to be quite stable with respect to the $\mathbf{k}$ sampling: only in the case of the poorest $\mathbf{k}$ mesh here adopted, $I S=16$, the $E L F$ peak appears noisy even if $\omega_{p}$ is correctly predicted with respect to the $I S=36$ reference.

- The DFT functionals.

SOS and SC-CP $E L F(\omega)$ and $R(\omega)$ spectra computed with several DFT functionals are reported in Figure 5. We first consider $E L F(\omega)$-SOS. The value of $\omega_{p}$ is $16.4,18.2,17.5$, 18.1 and $18.6 \mathrm{eV}$ for LDA, PBE, F10LYP, F15LYP and B3LYP, respectively. All the plasmon 
pole frequencies are shifted when SC-CP calculations are performed to $\omega_{p}=18.1,16.3,16.3$, 15.6 and $15.3 \mathrm{eV}$, respectively. With respect to the SOS counterpart, in SC-CP calculations, all functionals behave in a similar way: i) they do not alter dramatically the shape of the $E L F$ peak (it becomes less smooth with the appearance of shoulders that in some cases move to form a second peak); ii) they shift $\omega_{p}$. The plasmon energy is very sensitive to the rather flat plot of the real part of the dielectric constant when it crosses the frequency axis, and shoulders in the plot of $E L F(\omega)$ are related to oscillations in $\mathscr{R}_{e}\left(\varepsilon^{\infty}\right)$ as illustrated in Figure S2 of ESI where $\mathscr{R}_{e}\left(\varepsilon^{\infty}\right)$ is depicted in a narrow region close to $\varepsilon^{\infty}=0$.

We consider now $R(\omega)$. LDA and PBE SC-CP spectra are actually indistinguishable with respect to the corresponding SOS ones. With hybrid functional $R(\omega)$-CP spectra acquire more structure, especially in the $3-7 \mathrm{eV}$ region. We can also recognize a drop in $R$ in correspondence to the plasmon pole of $E L F$.

Computed $E L F(\omega)$ and $R(\omega)$ have been compared with the available experimental data. Concerning $E L F$, we can underline the good estimate of $\omega_{p}$ provided by PBE and F10LYP (the experimental value is $16.8 \mathrm{eV}^{38}$ ); however with PBE the plasmon energy corresponds to the less intense peak in the $\operatorname{ELF}(\omega)$ spectrum, whereas it is related to the main peak in the F10LYP case (see Fig. 5). A relatively good agreement with experiment (Ref. 39) is obtained for $R(\omega)$ : looking at the F10LYP spectrum we can identify a doublet in the $3.5-4 \mathrm{eV}$ region $(\mathrm{R} \geq 50 \%)$ and a peak at $5 \mathrm{eV}(\mathrm{R}=55 \%)$ before the principal one obtained at $7 \mathrm{eV}(\mathrm{R}=75 \%)$ while the experimental plot of $\mathrm{R}$ shows a first peak at $3.4 \mathrm{eV}(\mathrm{R} \simeq 60 \%)$, the largest one at $4.6 \mathrm{eV}(\mathrm{R} \geq 75 \%)$ and the beginning of a diffuse one at $7 \mathrm{eV}(\mathrm{R} \leq 70 \%)$. The relative intensities are not perfectly reproduced in our calculation with respect to the good experimental resolution in this range of energy, the overall shape is, however, very similar. In particular, we obtain $\mathrm{R}=30 \%$ as in experiment for $h v \leq 1 \mathrm{eV}$.

\section{SiC}

The cubic (3C) polytype of $\mathrm{SiC}$ ( $F \overline{4} 3 m$ space group) is a wide gap semi-conductor with a static dielectric constant twice smaller than that of Silicon (see Table 1), and an expected larger electron- 
hole interaction. The electronic structure, band structure BS and projected density of states PDOS are resumed in Figure S3 and Table S1 of ESI.

$\mathrm{SiC}$ has an indirect band gap $E_{g}(\Gamma \rightarrow X)$ at $2.54 \mathrm{eV}$, a direct band gap in $X, E_{g}(X)=5.87 \mathrm{eV}$ (F15LYP); considerably larger are the gaps computed at the other special k points: $E_{g}(L)=7.50$ $\mathrm{eV}, E_{g}(\Gamma)=7.21 \mathrm{eV}$ and $E_{g}(U(X))=7.13 \mathrm{eV}$; the $E_{H O M O \rightarrow L U M O+1}(\Gamma)=8.26 \mathrm{eV}$ is the other lowest energy difference between VB and CB bands, followed by $E_{H O M O \rightarrow L U M O+1}(X, L)=9.54,9.60 \mathrm{eV}$, Table S1 of ESI.

The UV-spectrum computed with F10LYP and F15LYP functionals is reported in Figure 6. The F15LYP UV-spectrum is dominated by the presence of two very intense peaks at 7.2 and $7.9 \mathrm{eV}$ and small ones at 5.9, 9.2 and $9.5 \mathrm{eV}$. The analysis of the $\mathrm{SiC}$ band structure (see Figure S2 and Table S1 of ESI) permits the following attributions: the lowest peak comes from $H O M O \rightarrow L U M O$ transitions in the vicinity of the minimum direct gap; the high peaks at 7.3 and $7.9 \mathrm{eV}$ from $H O M O \rightarrow L U M O$ transitions, close to the $L$ point the former and along the $\Gamma-K-W$ line the latter; the peak at $9.2 \mathrm{eV}$ to $H O M O \rightarrow L U M O+1$ transitions along the $\Gamma-L$ line and the 9.5 to $H O M O \rightarrow L U M O+1$ transitions close to $X$. Several experimental studies ${ }^{40,41}$ have analyzed the structure of $\mathscr{I}_{m}(\varepsilon)$ identifying peaks at 5.96, 7.43, 7.73, 9.03, and $9.4 \mathrm{eV}$. Positions and assignments of computed peaks are, therefore, in agreement with observations, see Ref. 41. The F15LYP computed spectrum reproduces quite nicely the experimental one, although slightly shifted towards lower energies (by about 0.1-0.2 eV) (see Figure 3); rather unsatisfactory is, instead, the F10LYP one, due to a reversed ratio between intensities of the two main peaks and a smoother shape close to the band gap edge, Figures 3 and 6.

The presence of a very weak bound exciton has been also found: the highly resolved F15LYP spectrum $(I S=150, \eta=0.01 \mathrm{mHa})$ computed in a narrow region close to the band gap edge shows a first resonance downshifted with respect to $E_{g}=5.873 \mathrm{eV}$ by $2.0 \mathrm{meV}$.

$R(\omega)$ and $E L F(\omega)$ CP and SOS spectra computed with PBE, F10LYP and F15LYP are reported in Figure 7. A good agreement with experiment ${ }^{42}$ is obtained for the PBE and F10LYP plasmon energies, at 21.7 and $21.9 \mathrm{eV}$, respectively (Table 1). 
For $R$, a value of $20 \%$ is obtained at low energy $(h v \leq 1 \mathrm{eV})$ in good agreement with the experimental datas of Logothetidis and Petalas. ${ }^{40}$ A shoulder around $6 \mathrm{eV}(R \simeq 40 \%)$ and a peak at 7-8 eV ( $R \leq 60 \%$ with PBE and F10LYP, $R>80 \%$ with F15LYP) are found with the coupled-perturbed calculation, also in agreement with the latter reference $(R=40 \%$ at $6 \mathrm{eV}$ and $R \simeq 60 \%$ at $7.8 \mathrm{eV})$. Above $12 \mathrm{eV}, R$ is essentially constant and equal to about $40-50 \%$ up to $18 \mathrm{eV}$ (CP-F15LYP) before decreasing to zero at higher energy. No experimental datas have been found for comparison in this range of energy.

\section{LiF}

$\mathrm{LiF}$ has a rock-salt structure $\left(F m \overline{3} m\right.$ space group); $\varepsilon^{\infty}=1.92$ (see Table 1$)$.

The electronic structure, band structure BS and projected density of states PDOS of LiF are shown in Figure S4 of ESI. At the $\Gamma$-point the gap $\left(E_{g}(\Gamma)\right)$ is equal to $15.39,14.07$ and $11.61 \mathrm{eV}$ for B3LYP, F40LYP and F50LYP, respectively (see Table 1); the experimental gap is $14.1-14.2 \mathrm{eV},{ }^{43}$ thus close

to the F40LYP value. The gap in correspondence to other special $\mathbf{k}$ points are: $E_{g}(L)=14.09 ; 16.61$; $17.95 \mathrm{eV}, E_{g}(X)=18.63 ; 21.34 ; 22.81 \mathrm{eV}$ (B3LYP; F40LYP;F50LYP).

The SC-CP F50LYP UV spectrum, Figure 8, is characterized by an intense peak at $13.86 \mathrm{eV}$, that is, $1.48 \mathrm{eV}$ lower than $E_{g}(\Gamma):$ it is due to bound excitons originated from $H O M O \rightarrow L U M O$ transitions in the vicinity of the minimum direct gap; from 15 to $25 \mathrm{eV}$ the spectrum is characterized by interband transitions from oscillators localized in a wide region along the $\Gamma-L-X$ path, see Figure S3 of ESI. By comparing the computed spectrum with the experimental one (Refs. 44,45) we see that, although the overall shape is well reproduced, the calculated exciton peak is shifted to higher energy (the experimental one is at $12.6 \mathrm{eV}$ ), Figure 3. With F40LYP the exciton is at $12.85 \mathrm{eV}$, shifted with respect to $E_{g}(\Gamma)$ by $1.25 \mathrm{eV}$ and in nice agreement with the position of the observed one. Unlike F50-F40LYP, the B3LYP spectrum appears unsatisfactory because of the absence of the bound exciton: we see, indeed, a small peak at $12.01 \mathrm{eV}$ and a more intense one at $13.5 \mathrm{eV}$, both at higher energy with respect to the band gap $\left(E_{g}(\Gamma)=11.61 \mathrm{eV}\right)$.

$R(\omega)$ shows a first thin peak $(R \simeq 65 \%)$ in the exciton energy range at about $12-14 \mathrm{eV}$ and a wide 
one $(R \simeq 50 \%)$ in the region between 20 and $28 \mathrm{eV}$, Figure 9. Both signals are experimentally observed, the former $(R \simeq 50 \%)$ at $11 \mathrm{eV}$ and the latter $(R \simeq 40 \%)$ centered at $23 \mathrm{eV}$, see Refs. 44,45 .

$E L F(\omega)$ spectra are reported in Figure 9. In all spectra we can recognize small losses in the exciton region and a wide intense one around $25 \mathrm{eV}$ due to plasmon resonance of valence electrons. The plasmon energies are at 25.3, 25.7 and 25.9 for B3LYP, F40LYP and F50LYP in good agreement with the experimental value, $\omega_{p}=24.9 \mathrm{eV}^{45,46}$ and $\omega_{p}=25.7 \mathrm{eV} .^{47}$

\section{Discussion}

$\mathscr{I}_{m}\left(\varepsilon^{\infty}\right)$ computed with the SC-CP procedure correctly reproduces the experimental UV spectrum and properly describes the observed excitons, weakly bound in $\mathrm{Si}$ and $\mathrm{SiC}$, strongly bound in $\mathrm{LiF}$. In the SC-CP scheme crystalline orbitals can relax in response to an electric field perturbation and excitons appear. Relaxation is ruled by the coupling matrices $U$, that in turn depend on the $B_{i a, \mathbf{k}}^{i a(a i), \mathbf{k}}$ integrals. This leads to a resonance frequency shifted with respect to the vertical transition $i_{\mathbf{k}} \rightarrow a_{\mathbf{k}}$ by the real quantity $B_{i a, \mathbf{k}}^{i a, \mathbf{k}}=2\left\langle i_{\mathbf{k}} a_{\mathbf{k}} \mid a_{\mathbf{k}} i_{\mathbf{k}}\right\rangle-\left\langle i_{\mathbf{k}} a_{\mathbf{k}} \mid i_{\mathbf{k}} a_{\mathbf{k}}\right\rangle$. Thus, in a certain region of the $\mathbf{k}$ space, generally in correspondence to a critical point, there may be an accumulation of oscillators resonating in a narrow energy range and whose strengths sum up. The result is the presence in the UV spectrum of new intense peaks (excitons) which are not observed in the SOS analogue. The number of oscillators $\rho\left(\omega_{i a, \mathbf{k}}\right)$ resonating approximately at $\omega_{i a, \mathbf{k}} \pm \eta$ depends on the DOS shape of the valence and conduction bands: if the latter ones are thin as in molecular systems (or for k-point where the effective mass is large), the density of transition states at the gap, multiplied by $\eta$, will be equal to one since there is only the $i_{\mathbf{k}} \rightarrow a_{\mathbf{k}}$ transition in the energy range $\omega_{i a, \mathbf{k}} \pm \eta$ ( $\mathbf{k}=\mathbf{0}$ for molecule), and the shift of the pole will be as large as $B_{i a, \mathbf{k}}^{i a, \mathbf{k}}$. On the contrary for semi-conductors with a small effective mass at the gap, $\rho\left(\omega_{i a, \mathbf{k}}\right)$ is small and the pole is not expected to be shifted a lot. However, for photon energy larger than the gap and corresponding to transition energies between VB and CB states belonging to the DOS maxima, the shift is the largest one. In other 
words, the shape of the UV-visible spectrum can change when the coupling is taken into account even if the gap (lowest transition energy) is unshifted. Nevertheless, the appearance of excitons in the UV spectrum is observed only when a certain amount of exact exchange is incorporated in the DFT functionals, whereas with pure DFT $\mathscr{I}_{m}\left(\varepsilon^{\infty}\right)$-CP and $\mathscr{I}_{m}\left(\varepsilon^{\infty}\right)$-SOS practically coincide. An explanation for this behavior is that the integral, $2\left\langle i_{\mathbf{k}}(1) a_{\mathbf{k}}(2)\left|\frac{1}{r_{12}}\right| a_{\mathbf{k}}(1) i_{\mathbf{k}}(2)\right\rangle$, the Coulomb term of $B_{i a, \mathbf{k}}^{i a, \mathbf{k}}$, is probably relatively small with respect to the integral $\left\langle i_{\mathbf{k}}(1) a_{\mathbf{k}}(2)\left|\frac{1}{r_{12}}\right| i_{\mathbf{k}}(1) a_{\mathbf{k}}(2)\right\rangle$, the HF exchange potential of $B_{i a, \mathbf{k}}^{i a, \mathbf{k}}$. In the "Coulomb term": $\left\langle i_{\mathbf{k}}(1) a_{\mathbf{k}}(2)\left|\frac{1}{r_{12}}\right| a_{\mathbf{k}}(1) i_{\mathbf{k}}(2)\right\rangle$ (as well as in $B_{i a, \mathbf{k}}^{a i, \mathbf{k}}$ ), electron (1) belongs to different and orthogonal $i_{\mathbf{k}}(1)$ - and $a_{\mathbf{k}}(1)$-orbitals, leading to a small value of the integral (at long distance $r_{12}$ ). On the contrary, the "exchange term" represents the average repulsion $1 / r_{12}$ between the hole $i_{\mathbf{k}}^{2}(1)$ and electron $a_{\mathbf{k}}^{2}(2)$ densities. This is the reason why the energy shift of the pole is generally negative, as shown in Application, when using HF or hybrid DFT hamiltonians $\left(B_{i a, \mathbf{k}}^{i a, \mathbf{k}}\right.$ is negative and a pole appears before the transition energy: $\left.\omega_{i a, \mathbf{k}}<\varepsilon_{a_{\mathbf{k}}}^{(0)}-\varepsilon_{i_{\mathbf{k}}}^{(0)}\right)$. In DFT, the integrals $\left\langle i_{\mathbf{k}} a_{\mathbf{k}} \mid i_{\mathbf{k}} a_{\mathbf{k}}\right\rangle$ coming from the HF exchange potential is missing and replaced by integrals similar to the "Coulomb term" multiplied by the derivative of the exchange-correlation functional of the electron density (and its gradient) with respect to the field. ${ }^{5,24}$ Then, the orbital relaxation effect in presence of a frequency-dependent field near resonances is weak, there is no shift of the energy $\left(B_{i a, \mathbf{k}}^{i a, \mathbf{k}}=2\left\langle i_{\mathbf{k}} a_{\mathbf{k}} \mid a_{\mathbf{k}} i_{\mathbf{k}}\right\rangle \simeq 0\right)$, and the lowest excitation energy is equal to the direct band gap, as it has been also shown in Refs. 24,25 for extended systems. In addition "pure" DFT suffers for the self interaction error, the spurious electrostatic interaction of an electron with itself. As a result, the electron is partly repelled from itself leading to an asymptotic decay of the exchange-correlation potential that is too weak. Correction for the self interaction error is necessary to properly describe the polarization of any system. It is partially embodied in the fraction of exact exchange in hybrids DFT, thus partially recovering the correct asymptotic behavior of exchange-correlation potential. ${ }^{25,48-50}$

The necessity to use hybrids to calculate optical transitions has been generally recognized, although, the best choice for the exchange-correlation functional is still debated. Conventional hybrids are not able to describe all excitons and especially those involving charge transfer excitations. Several 
functional forms have been proposed in order to circumvent this problem and among them we mention the use of a tuned fraction of exact exchange in a conventional hybrid functional; the use of semiempirical conventional hybrid functionals which contain a large number of empirical parameters with the aim of providing a balanced treatment of main properties including long-range charge transfer interactions; long-range corrected functionals with a fixed (even if empirically determined) long-range separation parameter; long-range separated functionals with system-specific optimal tuning to determine the range separation parameter, see Refs 20,21 and references therein. Although this latter procedure has been successfully applied for the description of polarizability and band gaps in solids, no comprehensive applications to the calculations of the whole $U V, E L F$ and $R$ spectra has been reported yet. Kresse and coworkers applied the HSE functionals (varying also the range separation parameter) to semiconductors and insulators: however they were not able to satisfactorily describe the LiF absorption spectrum. ${ }^{51}$ Therefore we preferred to follow the former scheme that appears also particularly attracting, since it involves the use of conventional exchange functionals, whose performances have been well assessed during decades of studies and applications. To this extent, in the spirit of the work of Marques et al., ${ }^{18}$ Skone et al., ${ }^{19}$ Brothers et al., ${ }^{22}$ we found that a percentage of HF-exchange corresponding to the inverse of the static optical dielectric constant, $1 / \varepsilon^{\infty}(0)$ (around $10 \%$ for $\mathrm{Si}, 15-20 \%$ for $\mathrm{SiC}$ and $40-50 \%$ for $\mathrm{LiF}$ ), leads to band gaps and static dielectric constants in good agreement with experiment. It follows also that the UV-visible absorption spectrum (position and relative intensity of the peaks) is also well described with these percentage values, particularly for SiC (Figure 3). A little bit less straightforward is the case of Si for which the F10LYP functional, the best choice according to this procedure, does not give a UV spectrum as good as the F15LYP. Also in the case of LiF we found a better result with F40LYP instead of F50LYP, the best choice according to this scheme. This finding is not surprising since the procedure leads to a simple description of the screened electron repulsion in a medium by empirically tuning the amount of exact exchange with only one parameter (the inverse of the static dielectric constant of the crystal itself). The choice of this unique parameter may be questionable. In addition to this attempt to obtain a satisfactory description of a physical property 
(as the dielectric constant) by changing the amount of exact exchange can alter the delicate balance between exchange and correlation in ways that could be disadvantageous for other properties. Nevertheless, our work shows that a fraction of exact exchange approximatively equal to the inverse of the static dielectric constant leads to UV spectra comparable with the experimental ones. If no shift of the $\mathrm{CB}$ is found for semiconductors like $\mathrm{Si}$ and medium $\mathrm{SiC}$, the relative intensity is enhanced at low transition energy after the electron gap. As regards LiF, the "red" shift of the optical gap with respect to the lowest vertical transition energy increases as the percentage of HF-exchange (x-value) increases in the SC-CP process. However, this is also the case of the opposite "blue" shift of the lowest vertical transition energy, so that there is no simple linear relation between the optical gap value and $\mathrm{x}$, as for $\mathrm{Si}$ and $\mathrm{SiC}$, and more generally between the full spectrum $(U V, E L F$ and $R$ ) and the exchange percentage. Nevertheless, for the three studied compounds, the lowest energy peaks are enhanced by coupled hybrid methods with respect to SOS, leading to a shift of the whole absorption spectrum, as well as to a shift of ELF and $R$, towards low energies. As regards the plasmon energy, its value is very sensitive to small amplitude oscillations of $\mathscr{R}_{e}\left(\varepsilon^{\infty}\right)$ around zero; the ELF maximum can be shifted and shoulders appear. The origin of such oscillations comes from faked occupied to virtual vertical transitions that are not completely smoothed out by the SC-CP method. The ELF plasmon frequency is, however, relatively well described when the percentage value of exchange is around $10 \%$ for both $\mathrm{Si}$ and $\mathrm{SiC}$ crystals, and $40 \%$ for LiF. We have also shown that the band energy for which $\mathrm{Si}$ and $\mathrm{SiC}$ reflect the light (band energy where the reflectivity is not null) slightly decreases with the increasing percentage of HF-exchange, while the peak of $R$ at low energy becomes larger. This result was previously found for the absorption spectrum and showing that the SC-CP method involving virtual orbitals (as SCF occupied ones) in the optimization process leads to the decrease of the conduction band width. Virtual unrelaxed orbitals which can have very large unphysical eigenvalues finally do not contribute to the spectrum anymore, or at much lower energy than their eigenvalues, when they are included in the SC-CP process. 


\section{Conclusions}

In this work, the frequency-dependent coupled-perturbed method already implemented in the CRYSTAL code which uses localized atomic orbitals has been extended in order to compute the real and imaginary parts of the dielectric response property of periodic systems in the whole UVvisible range of energy. The use of the vectorial epsilon-algorithm has been essential to improve convergence of the SC-CP process near resonances. The method allows to obtain numerically both the poles and oscillator strengths of the electronic spectrum from the dielectric reponse property without storing large hamiltonian matrices to be diagonalized.

The percentage of Hartree-Fock exchange in the hamiltonian (with $100 \%$ of HF-exchange the method is equivalent to TDHF, with $0 \%$ to TDDFT) is easily modified and, its influence on the UV-visible absorption spectrum, electron loss function and reflectivity explored.

In this first application of the CRYSTAL code to the study of the excited states of solids we employed the well-known B3LYP hybrid functional: we have found that, incorporating a percentage of HF-exchange corresponding to the inverse of the static optical dielectric constant, $1 / \varepsilon^{\infty}(0)$, in the functional (used in Refs. 18,19 to obtain band gaps in good agreement with experiment), leads also to satisfactory UV-visible absorption spectra (position and relative intensity of the peaks). Delocalized, weakly bound excitons as in $\mathrm{Si}$, and localized, strongly bound ones as in $\mathrm{LiF}$, are both correctly described with this approach. The electron loss function and reflectivity computed by roughly employing the same amount of HF-exchange are also in good agreement with experiments.

\section{Acknowledgments}

The authors are grateful to Prof. R. Dovesi for kind help and fruitful suggestions. A.M. F. acknowledges CINECA (Iscra project B HOME) for computational resources. 


\section{Supporting Information}

Details about electron structures (DOS and band structures) togheter with plots of $\mathscr{R}_{e}\left(\varepsilon^{\infty}\right)$ in a narrow region close to $\varepsilon^{\infty}=0$ are provided as supplemantary information. This material is available free of charge via the Internet at http://pubs.acs.org. 


\section{References}

(1) Dovesi, R.; Saunders, V. R.; Roetti, C.; Orlando, R.; Zicovich-Wilson, C. M.; Pascale, F.; Civalleri, B.; Doll, K.; Harrison, N. M.; Bush, I. J. et al. CRYSTAL14 User's Manual, University of Torino 2014,

(2) Hurst, G.; Dupuis, M.; Clementi, E. Ab initio Analytic Polarizability, First and Second Hyperpolarizabilities of Large Conjugated Organic Molecules: Applications to Polyenes C4H6 to C2 2H2 4. J. Chem. Phys. 1988, 89, 385-395.

(3) Ferrero, M.; Rérat, M.; Orlando, R.; Dovesi, R. The Calculation of Static Polarizabilities of 1-3D Periodic Compounds. the Implementation in the Crystal Code. J. Comp. Chem. 2008, 29, $1450-1459$.

(4) Dovesi, R.; Orlando, R.; Zicovich-Wilson, C. M.; Civalleri, B.; Maschio, L.; Erba, A.; Casassa, S.; Ferrabone, M.; Pierre, M. D. L.; D’Arco, P. et al. CRYSTAL14: A Program for the Ab Initio Investigation of Crystalline Solids. Int. J. Quant. Chem. 2014, 114, 1287-1317.

(5) Ferrero, M.; Rérat, M.; Orlando, R.; Dovesi, R.; Bush, I. J. Coupled Perturbed Kohn-Sham Calculations of Static Polarizabilities of Periodic Compounds. J. Phys. Conf. Series 2008, 117, 12016.

(6) Bernasconi, L.; Tomić, S.; Ferrero, M.; Rérat, M.; Orlando, R.; Dovesi, R.; Harrison, N. M. First-Principles Optical Response of Semiconductors and Oxide Materials. Phys. Rev. B 2011, $83,195325$.

(7) Erba, A.; Dovesi, R. Photoelasticity of Crystals from Theoretical Simulations. Phys. Rev. B 2013, $88,045121-8$.

(8) Mahmoud, A.; Erba, A.; El-Kelany, K. E.; Rérat, M.; Orlando, R. Low-Temperature Phase of BaTiO3: Piezoelectric, Dielectric, Elastic, and Photoelastic Properties from ab Initio Simulations. Phys. Rev. B 2014, 89, 045103-9. 
(9) Karna, S. P.; Dupuis, M. Frequency Dependent Nonlinear Optical Properties of Molecules: Formulation and Implementation in the HONDO program. J. Comp. Chem. 1991, 12, 487-504.

(10) Lorenz, M.; Usvyat, D.; Schütz, M. Local ab initio Methods for Calculating Optical Band Gaps in Periodic Systems. I. Periodic Density Fitted Local Configuration Interaction Singles Method for Polymers. J. Chem. Phys. 2011, 134, 094101-14.

(11) Lorenz, M.; Maschio, L.; Schütz, M.; Usvyat, D. Local Ab Initio Methods for Calculating Optical Band Gaps in Periodic Systems. II. Periodic Density Fitted Local Configuration Interaction Singles Method for Solids. J. Chem. Phys. 2012, 137, 204119-14.

(12) Olevano, V.; Reining, L. Excitonic Effects on the Silicon Plasmon Resonance. Phys. Rev. Lett. 2001, 86, 5962.

(13) Rohlfing, M.; Louie, S. G. Electron-Hole Excitations in Semiconductors and Insulators. Phys. Rev. Lett. 1998, 81, 2312-2315.

(14) Aspnes, D. E.; Studna, A. A. Dielectric functions and optical parameters of Si, Ge, GaP, GaAs, GaSb, InP, InAs, and InSb from 1.5 to 6.0 eV. Phys. Rev. B 1983, 27, 985-1009.

(15) Philipp, H. R.; Ehrenreich, H. Optical Properties of Semiconductors. Phys. Rev. 1963, 129, $1550-1560$.

(16) Lautenschlager, P.; Garriga, M.; Cardona, M. Temperature Dependence of the Dielectric Function and Interband Critical Points in Silicon. Phys. Rev. B 1987, 36, 4821-4830.

(17) Onida, G.; Reining, L.; Rubio, A. Electronic Excitations: Density-Functional versus ManyBody GreenĂŹs-Function Approaches. Rev. Mod. Phys. 2002, 74, 601-659.

(18) Marques, M. A. L.; Vidal, J.; Oliveira, M. J. T.; Reining, L.; Botti, S. Density-Based Mixing Parameter for Hybrid Functionals. Phys. Rev. B 2011, 83, 035119-5.

(19) Skone, J. H.; Govoni, M.; Galli, G. Self-Consistent Hybrid Functional for Condensed Systems. Phys. Rev. B. 2014, 89, 195112-12. 
(20) Kronik, L.; Stein, T.; Refaely-Abramson, S.; Baer, R. Excitation Gaps of Finite-Sized Systems from Optimally Tuned Range-Separated Hybrid Functionals. J. Chem. Theory and Comp 2012, $8,1515-1531$.

(21) Eisenberg, H. R.; Baer, R. A New Generalized Kohn-Sham Method for Fundamental BandGaps in Solids. Phys. Chem. Chem. Phys. 2009, 11, 4674-4680.

(22) Brothers, E. N.; Izmaylov, A. F.; Scuseria, G. E.; Kudin, K. N. Analytically Calculated Polarizability of Carbon Nanotubes: Single Wall, Coaxial, and Bundled Systems. J. Phys. Chem. C 2008, 112, 1396-1400.

(23) Izmaylov, A. F.; Brothers, E. N.; Scuseria, G. E. Linear-scaling calculation of static and dynamic polarizabilities in Hartree-Fock and density functional theory for periodic systems. $J$. Chem. Phys. 2006, 125, 224105-9.

(24) Izmaylov, A. F.; Scuseria, G. E. Why are Time-Dependent Density Functional Theory Excitations in Solids Equal to Band Structure Energy Gaps for Semilocal Functionals, and How Does Nonlocal Hartree-Fock-type Exchange Introduce Excitonic Effects? J. Chem. Phys. 2008, 129, 034101.

(25) Hirata, S.; Head-Gordon, M.; Bartlett, R. J. Configuration Interaction Singles, Time-Dependent HartreeâĂŞFock, and Time-Dependent Density Functional Theory for the Electronic Excited States of Extended Systems. J. Chem. Phys. 1999, 111, 10774.

(26) Otto, P. Calculation of the Polarizability and Hyperpolarizabilities of Periodic Quasi-onedimensional Systems. Phys. Rev. B 1992, 45, 10876-10885.

(27) Rérat, M.; Ferrero, M.; Amzallag, E.; Baraille, I.; Dovesi, R. Comparison of the Polarizability of Periodic Systems Computed by Using the Length and Velocity Operators. J. Phys. Conf. Series 2008, 117, 012023. 
(28) Rice, J. E.; Amos, R. D.; Colwell, S. M.; Handy, N. C.; Sanz, J. Frequency Dependent Hyperpolarizabilities with Application to Formaldehyde and Methyl Fluoride. J. Chem. Phys. 1990, 93, 8828-8839.

(29) Dirac, P. A. M. Note on Exchange Phenomena in the Thomas-Fermi Atom. Proc. Cambridge Phil. Soc. 1930, 26, 376.

(30) Vosko, S. H.; Wilk, L.; Nusair, N. Accurate Spin-Dependent Electron Liquid Correlation Energies for Local Spin Density Calculations: A Critical Analysis. Can. J. Phys. 1980, 58, 1200.

(31) Perdew, J. P.; Burke, K.; Ernzehof, M. Generalized Gradient Approximation Made Simple. Phys. Rev. Lett. 1996, 77, 3865-3868.

(32) Becke, A. D. Density-Functional Exchange-Energy Approximation with Correct Asymptotic Behavior. Phys. Rev. A 1988, 38, 3098-3100.

(33) Lee, C.; Yang, W.; Parr, R. G. Development of the Colle-Salvetti Correlation-Energy Formula into a Functional of the Electron Density. Phys. Rev. B 1988, 37, 785-789.

(34) CRYSTAL14, website. http://www.crystal.unito.it/basis-sets.php.

(35) Durand, E. Solutions Numériques des Equations Algébriques, I\&II. Paris 1960,

(36) Wynn, P. Acceleration Techniques for Iterated Vector and Matrix Problems. Math. Comp. 1962, 16, 301-322.

(37) Brezinski, C. Some Results and Applications Abouts the Vector $\varepsilon$-Algorithm. J. of Math. 1974, 4,335 .

(38) Siebling, J. Optische Eigenschaften des Einkristallinen Siliziums aus Elektronenenergieverlustmessungen. Z. Phys. B 1978, 31, 355. 
(39) Green, M. A. Self-Consistent Optical Parameters of Intrinsic Silicon at 300 K Including Temperature Coefficients. Solar Energy Materials \& Solar Cells 2008, 92, 1305.

(40) Logothetidis, S.; Petalas, J. Dielectric Function and Reflectivity of 3CâĂŞSilicon Carbide and the Component Perpendicular to the c Axis of 6HâĂŞSilicon Carbide in the Energy Region 1.5-9.5 eV. J. Appl. Phys. 1996, 80, 1768.

(41) Theodorou, G.; Tsegas, G.; Kaxiras, E. Theory of Electronic and Optical Properties of 3C-SiC. J. Appl. Phys. 1999, 85, 2179.

(42) Heidt, A.; Chen, T.; Finger, F.; Rau, U.; Mayer, J.; Luysberg, M. Structure and Electronic Properties of $\mu \mathrm{c}-\mathrm{SiC}: \mathrm{H}$ for Photovoltaic Applications. J. Phys. Conf. Series 2011, 326, 012019.

(43) Kunz, A. B. Study of the Electronic Structure of Twelve Alkali Halide Crystals. Phys. Rev. B 1982, 26, 2056.

(44) Roessler, D. M.; Walker, W. C. Electronic Spectrum of Crystalline Lithium Fluoride. J. Phys. Chem. Solids 1967, 28, 1507-1515.

(45) Roessler, D. M.; Walker, W. C. Optical Constants of Magnesium Oxide and Lithium Fluoride in the Far Ultraviolet. J. Opt. Soc. Am. 1967, 57, 835-836.

(46) Caliebe, W. A.; Soininen, J. A.; Shirley, E. L.; Kao, C.-C.; Hamalainen, K. Phys. Rev. Lett. 2004, 84, 3907-3910.

(47) Creuzburg, M. Energieverlustspektren der Alkalihalogenide und der Metalle Cu, Ag und Au und Vergleich mit Optischen Messungen. Z. Phys. 1966, 196, 433.

(48) Perdew, J. P.; Zunger, A. Self-Interaction Correction to Density-Functional Approximations for Many-Electron Systems. Phys. Rev. B 1981, 23, 5048-5079.

(49) Pemmaraju, C. D.; Sanvito, S.; Burke, K. Polarizability of molecular chains: A self-interaction correction approach. Phys. Rev. B 2008, 77, 121204(R)-4. 
(50) van Leeuwen, R.; Baerends, E. J. Exchange-Correlation Potential with Correct Asymptotic Behavior. Phys. Rev. A 1994, 49, 2421-2431.

(51) Paier, J.; Marsman, M.; Kresse, G. Dielectric Properties and Excitons for Extended Systems from Hybrid Functionals. Phys. Rev. B 2008, 78, 121201(R)-4.

(52) Zucca, R. R. L.; Shen, Y. R. Wavelength-Modulation Spectra of Some Semiconductors. Phys. Rev. B 1970, 1, 2668-2676.

(53) Weast, R. C. Handbook of Chemistry and Physics 1989,

(54) Dalven, R. Temperature Coefficient of the Energy Gap of $\beta$-Silicon Carbide. J. Phys. Chem. Solids 1965, 26, 439.

(55) Nishino, S.; Powell, J. A.; Will, H. A. Production of Large-Area Single-Crystal Wafers of Cubic SiC for Semiconductor Devices. Appl. Phys. Lett. 1983, 42, 460.

(56) Li, Y.; Lin-Chung, P. J. Band Structure and Electronic Properties of Native Defects in Cubic SiC. Phys. Rev. B 1987, 36, 1130-1135.

(57) Lyle, P.; Choyke, W. J. Static Dielectric Constant of SiC. Phys. Rev. B 1970, 2, 2255-2256.

(58) Burstein, E.; Perkowitz, S.; Brodsk, M. H. The Dielectric Properties of the Cubic IV-VI Compound Semiconductors. J. de Physique Colloque C4 1968, 29, 78-83. 
Table 1: Electronic direct gap (in eV), coupled perturbed static (high-frequency) dielectric constant and plasmon energy (in eV) of $\mathrm{Si}, \mathrm{SiC}$ and $\mathrm{LiF}$.

\begin{tabular}{l|ccccccc} 
& PBE & F10LYP & F15LYP & B3LYP & F40LYP & F50LYP & Exp. \\
\hline $\mathrm{Si}$ & & & & & & & \\
Gap & 2.57 & 3.24 & 3.52 & 3.77 & & - & $3.4^{a}$ \\
$\varepsilon^{\infty}$ & 11.6 & 11.2 & 10.6 & 10.2 & - & & $11.8^{b}$ \\
Plasmon & 16.3 & 16.3 & 15.5 & 15.3 & - & & $16.8^{c}$ \\
\hline $\mathrm{SiC}$ & & & & & & & \\
Gap & 4.49 & 5.47 & 5.87 & 6.26 & - & & $6.0^{d}$ \\
$\varepsilon^{\infty}$ & 6.8 & 6.7 & 6.4 & 6.3 & - & & $6.5^{e}$ \\
Plasmon & 21.9 & 21.7 & 21.6 & 20.8 & - & & $22.2^{f}$ \\
\hline LiF & & & & & & & \\
Gap & 9.15 & 10.42 & 11.03 & 11.78 & 14.21 & 15.27 & $14.1^{g}$ \\
$\varepsilon^{\infty}$ & 2.0 & 1.9 & 1.9 & 1.8 & 1.8 & 1.7 & $1.9^{h}$ \\
Plasmon & 24.4 & 24.4 & 24.6 & 25.3 & 25.7 & 25.9 & $24.9^{i}{ }^{i} 25.7^{j}$ \\
\hline
\end{tabular}

a: Ref. 52 b: Ref. 53, c: Ref. 38, d: Ref. 54-56, e: Ref. 57, f: Ref. 42. g: Ref. 43 h: Ref. 58 i: Ref. 44,46 j: Ref. 47 

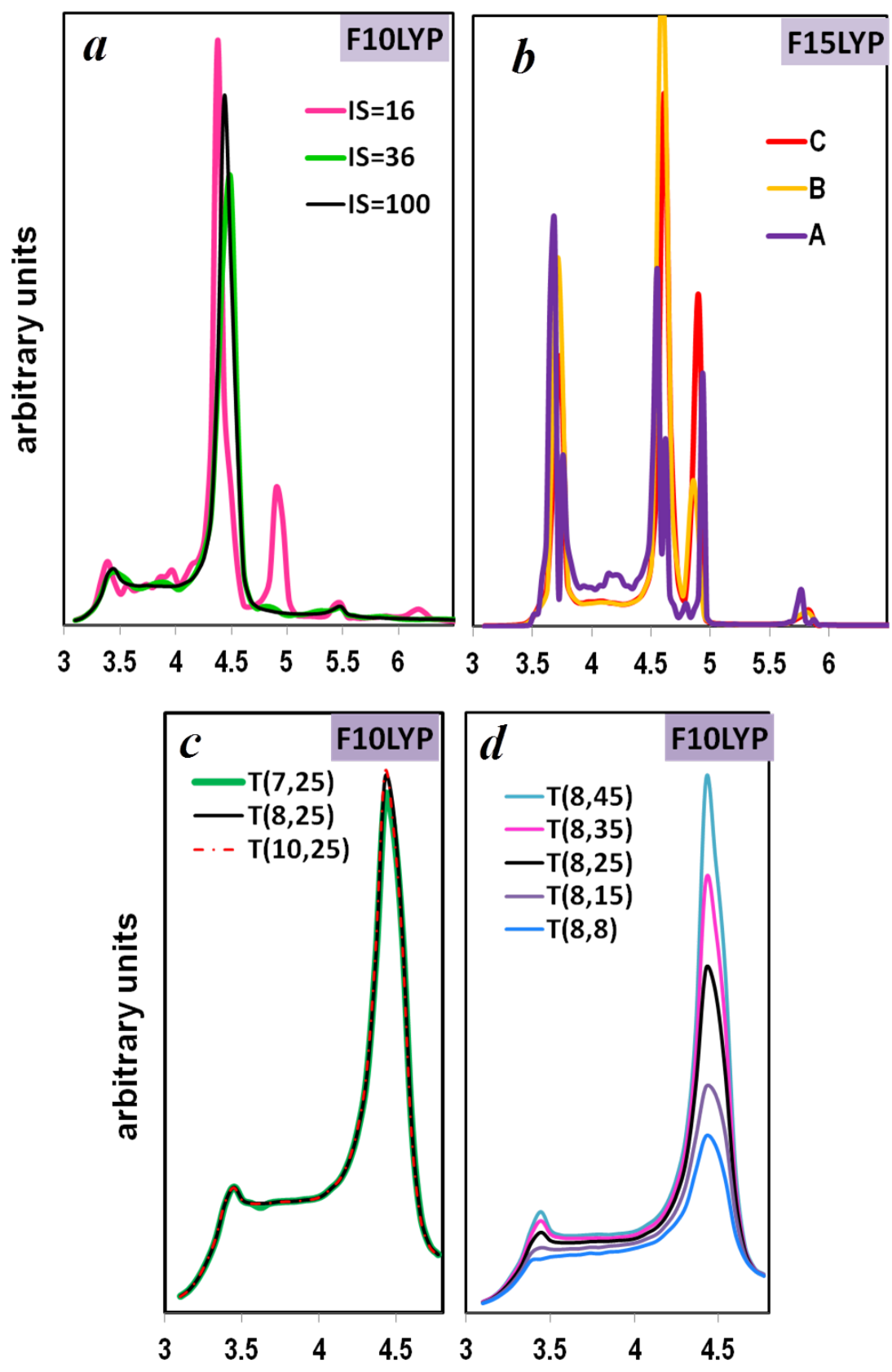

Figure 1: Effect of computational parameters on the imaginary part of the dielectric constant of Silicon (SC-CP calculation): a) reciprocal space sampling, $I S,(\mathrm{~T}=(10,25)$ and $\eta=0.055 \mathrm{eV})$; b): damping factor $\eta: \eta=0.055$ (A), 0.082 (B), 0.11 (C) eV, (T=10,25 and $I S=100)$; c-d): tolerances controlling the Coulomb and exchange series, $T,(I S=100$ and $\eta=0.055 \mathrm{eV})$ 


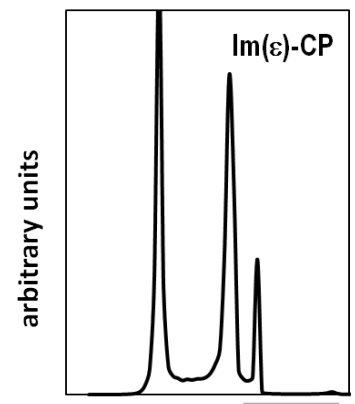

B3LYP

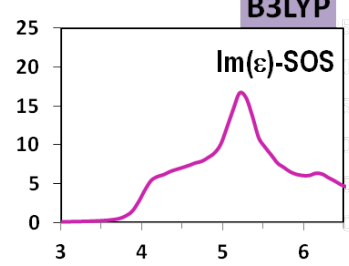

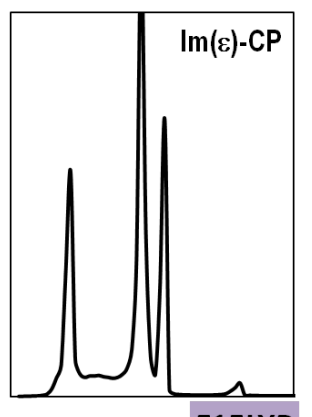

F15LYP

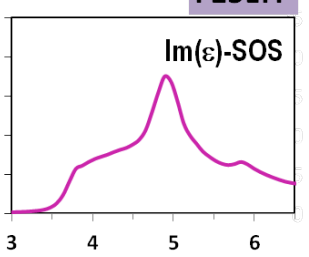

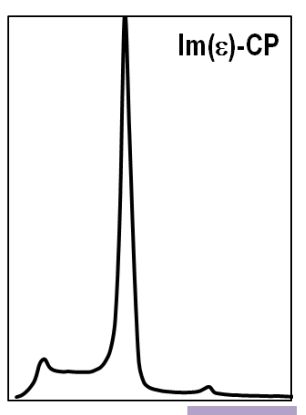
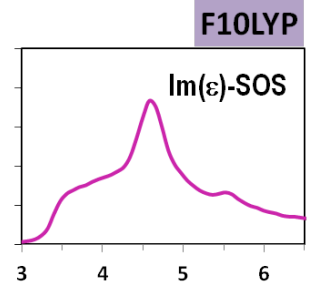
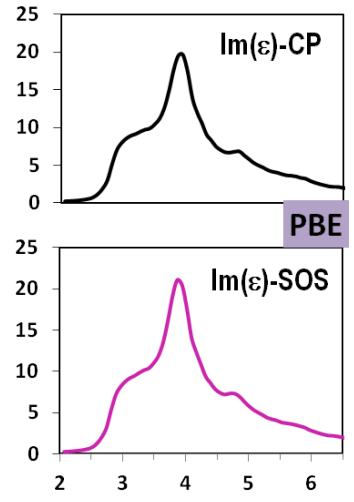
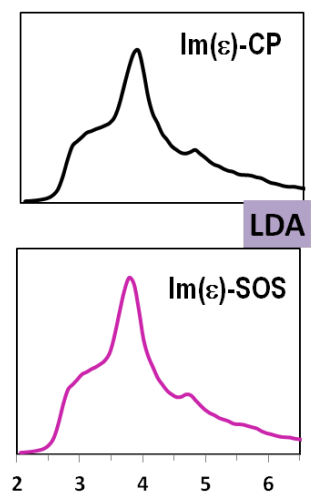

Figure 2: UV spectrum of Silicon computed with different functionals. $I S=100, T=(10,25)$ and $\eta=0.055 \mathrm{eV}$. 

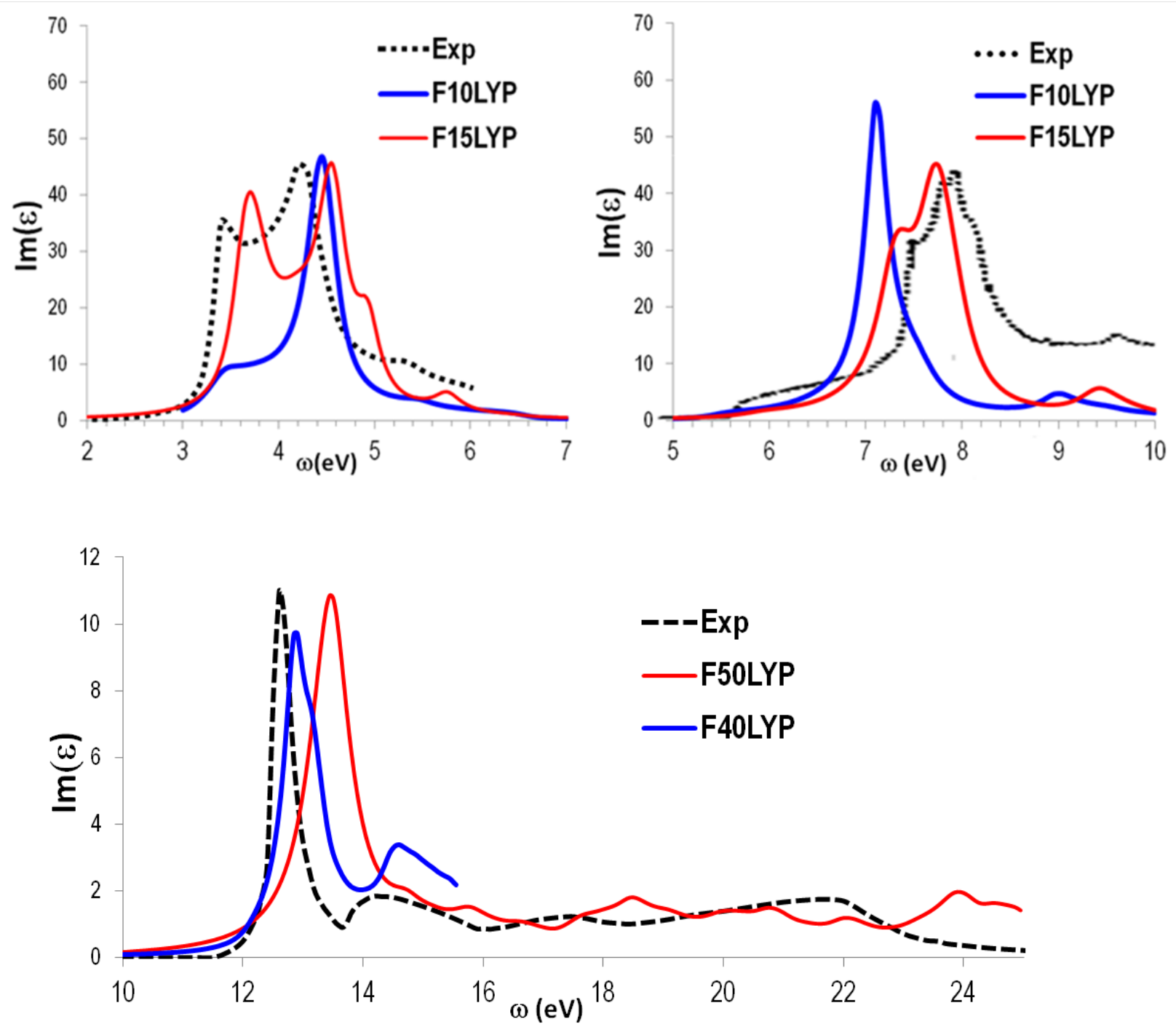

Figure 3: Computed and observed UV spectra of $\mathrm{Si}, \mathrm{SiC}$ and $\mathrm{LiF} . \mathscr{I}_{m}(\varepsilon)$-CP is obtained by normalizing and broadening the calculated spectrum with a lorenztian function. Experimental spectra from Refs. $14,41,45$. 


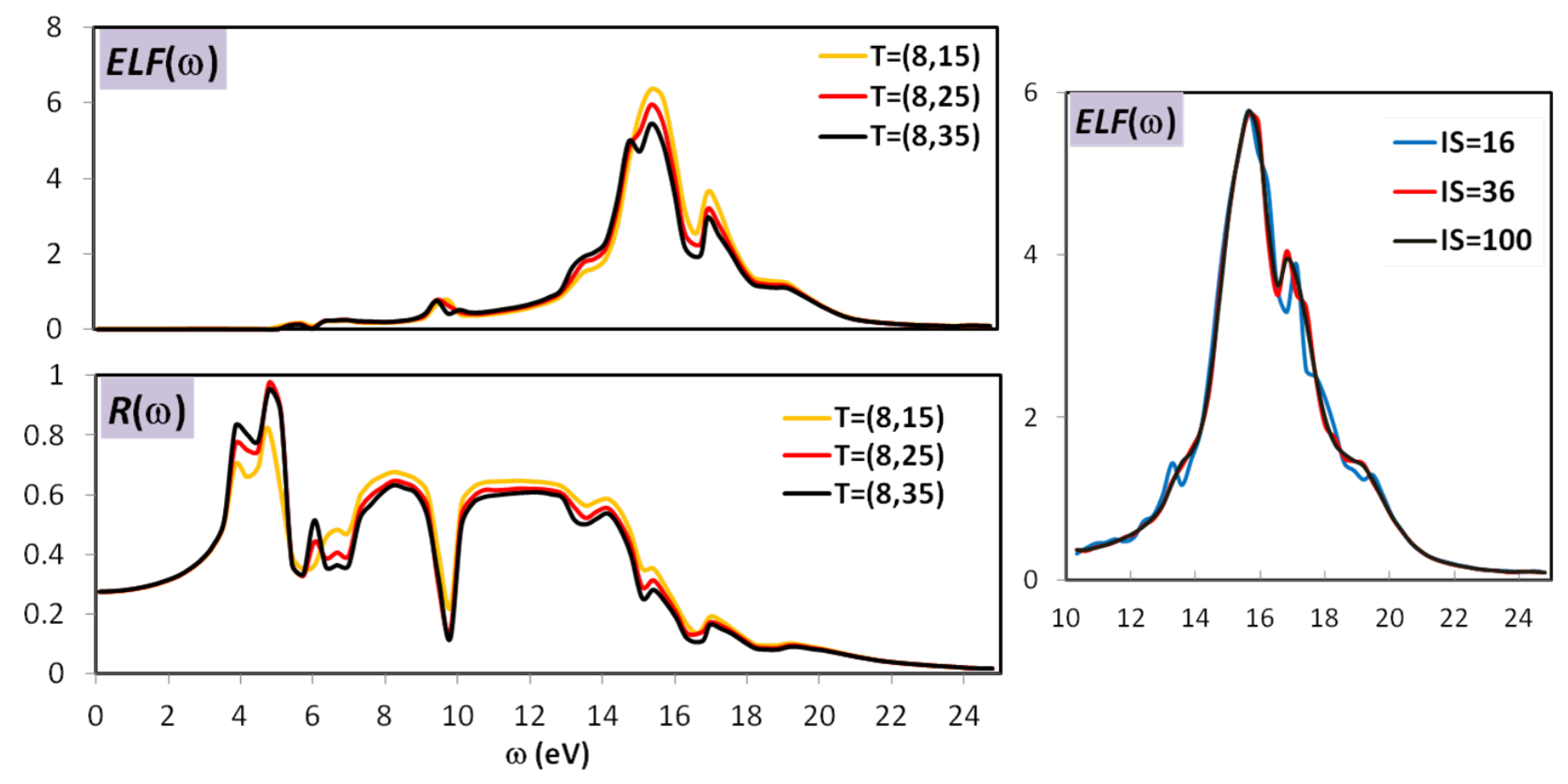

Figure 4: $E L F(\omega)$ and $R(\omega)$ of Silicon at the SC-CP B3LYP level of calculation: effect of $I S$ ( $\mathrm{T}=10,25$ and $\eta=0.11 \mathrm{eV}$ ) (right panel) and $T$ ( $I S=100$ and $\eta=0.11 \mathrm{eV}$ ) (left panels). 

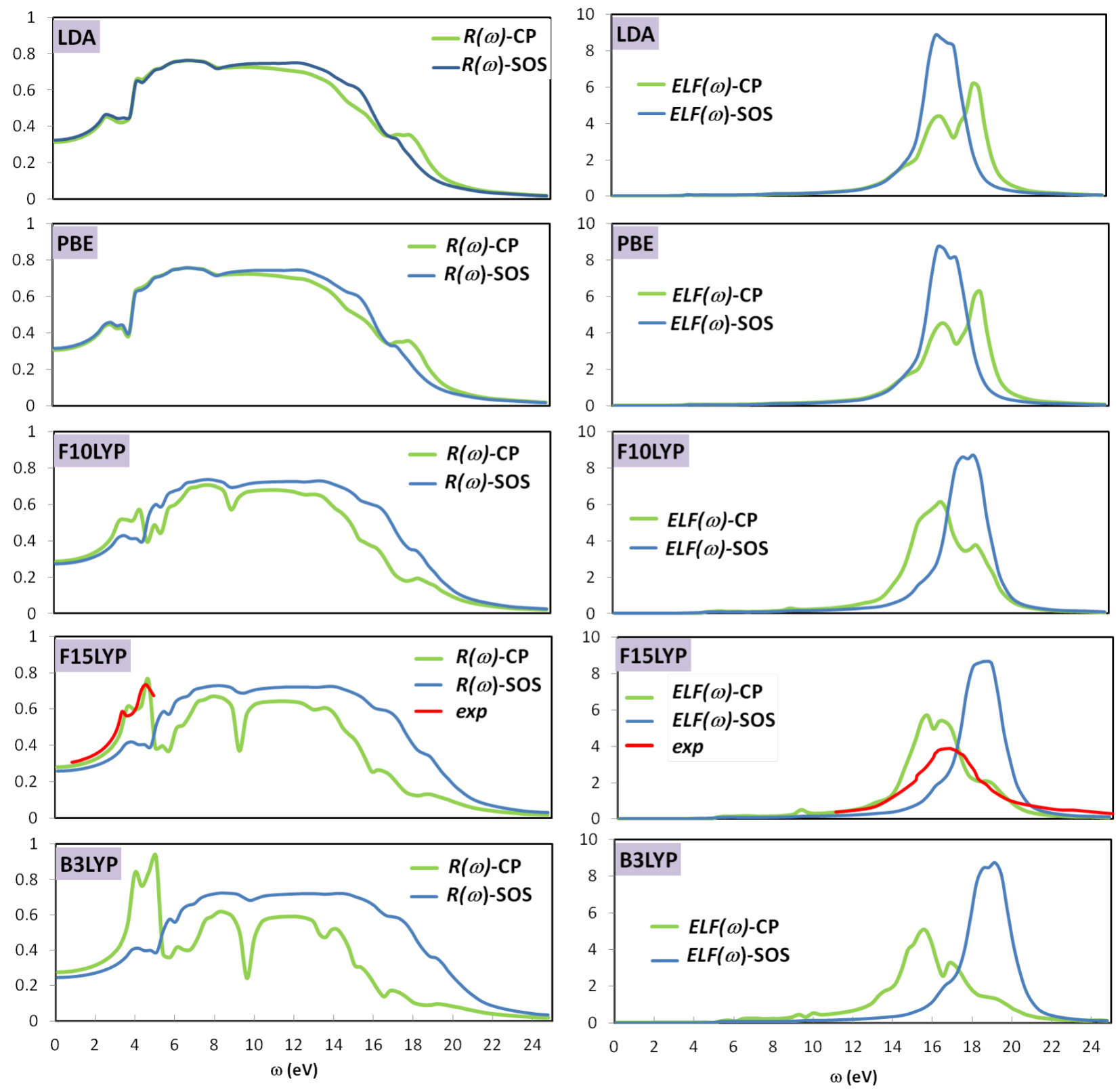

Figure 5: $E L F(\omega)$ and $R(\omega)$ of Silicon computed with different functionals. $I S=36, T=(10,25)$ and $\eta=0.11 \mathrm{eV}$. Experimental data from Refs 14,38 

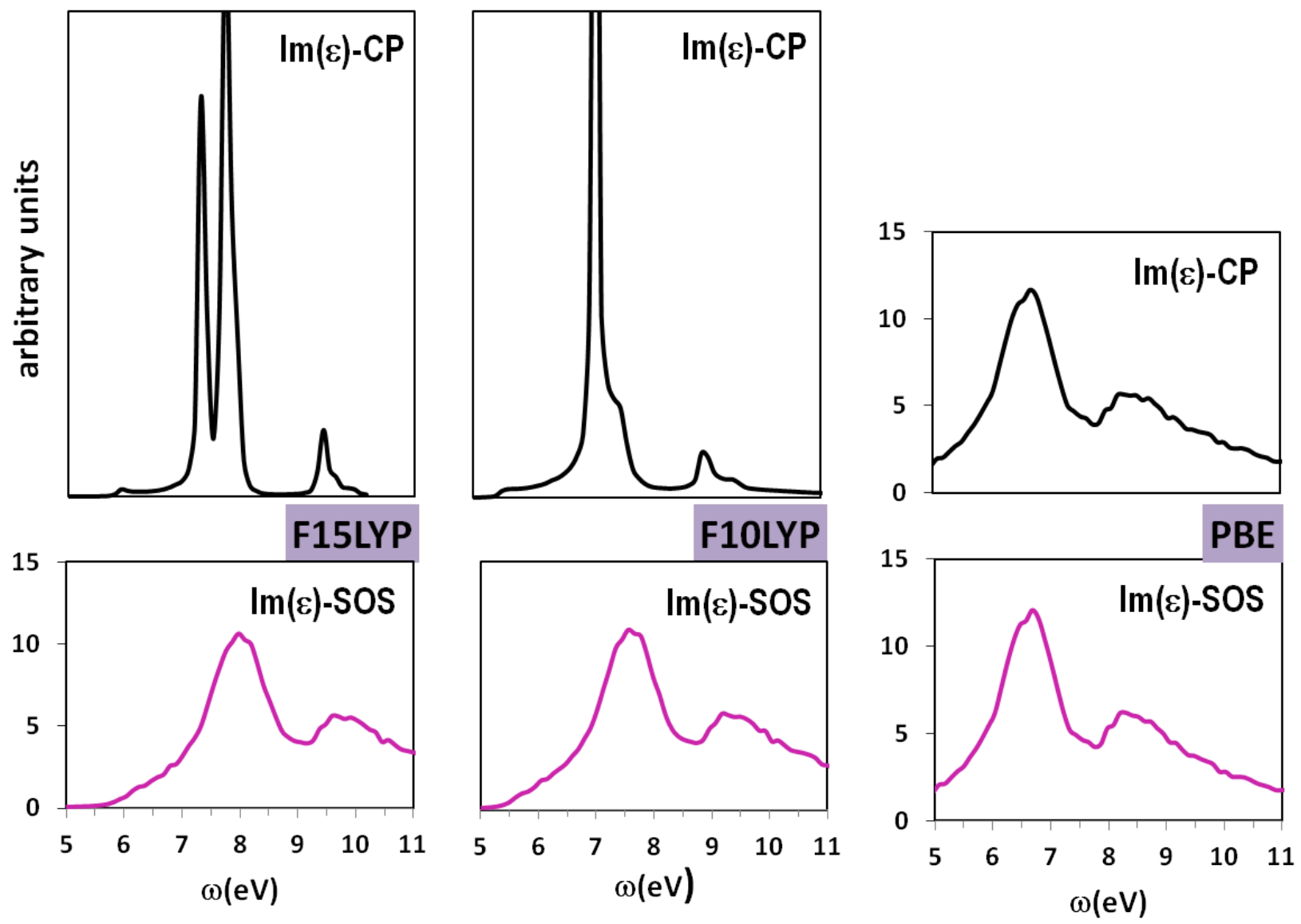

Figure 6: UV spectrum of $\mathrm{SiC}$ at various levels of theory. $I S=36, T=(8,25)$ and $\eta=0.055 \mathrm{eV}$. 

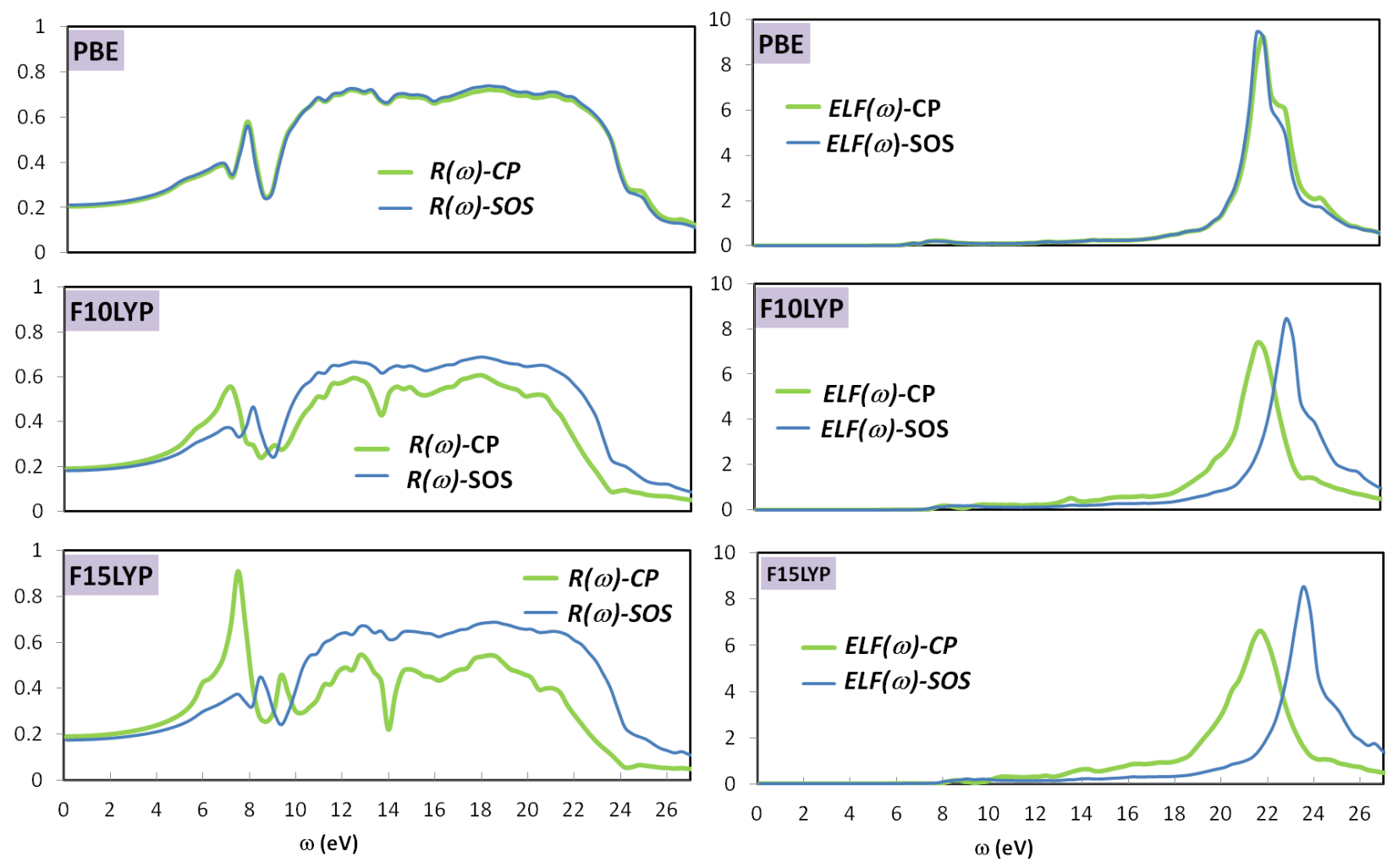

Figure 7: $E L F(\omega)$ and $R(\omega)$ of SiC: effect of the coupling at various levels of theory. IS=36, $T=(8,25)$ and $\eta=0.11 \mathrm{eV}$. Experimental data from Ref. 41 

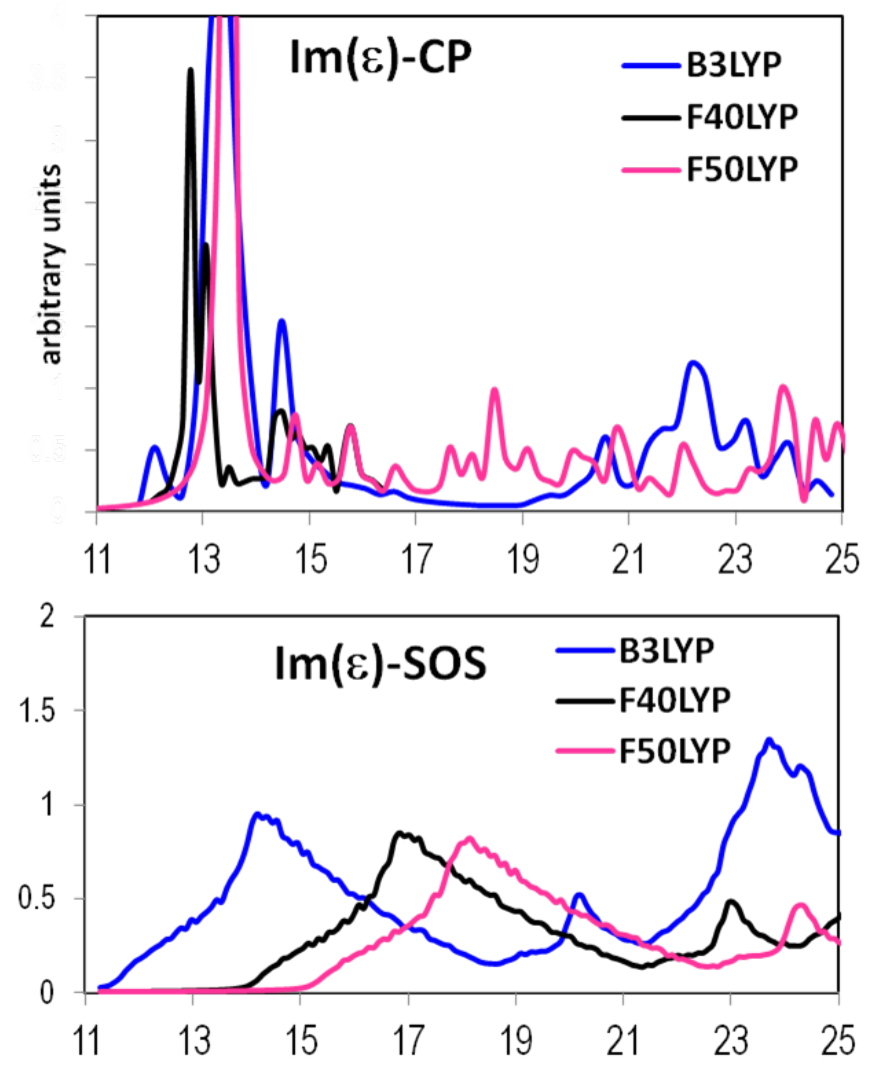

Figure 8: UV spectrum of $\mathrm{LiF}$ at various levels of theory. $I S=16 T=(8,25)$ and $\eta=0.055 \mathrm{eV}$. 

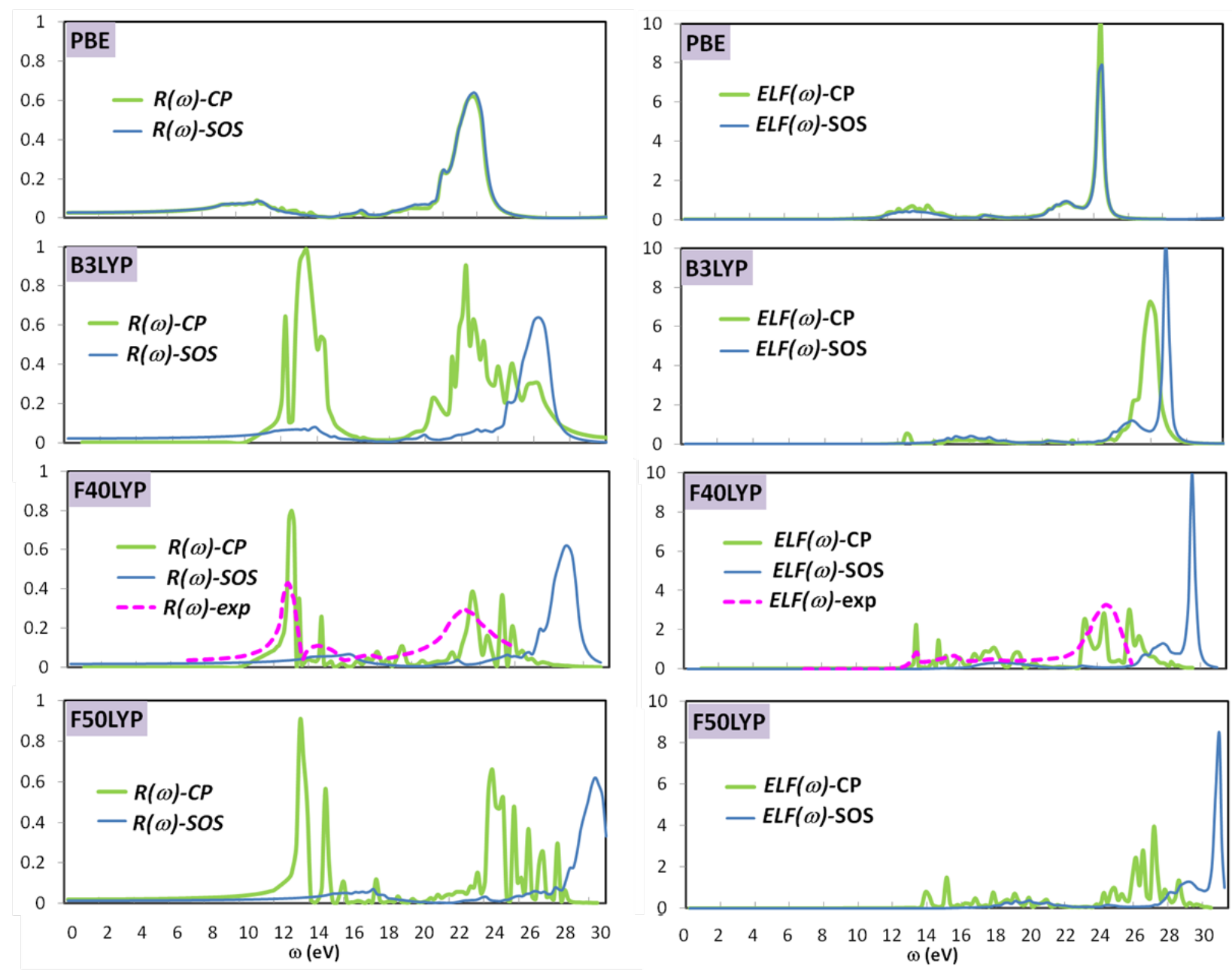

Figure 9: $E L F(\omega)$ and $R(\omega)$ of $\mathrm{LiF}$ at various levels of theory. $I S=16 T=(8,15)$ and $\eta=0.11 \mathrm{eV}$. Experimental data from Ref. 45 


\section{Graphical Abstract}

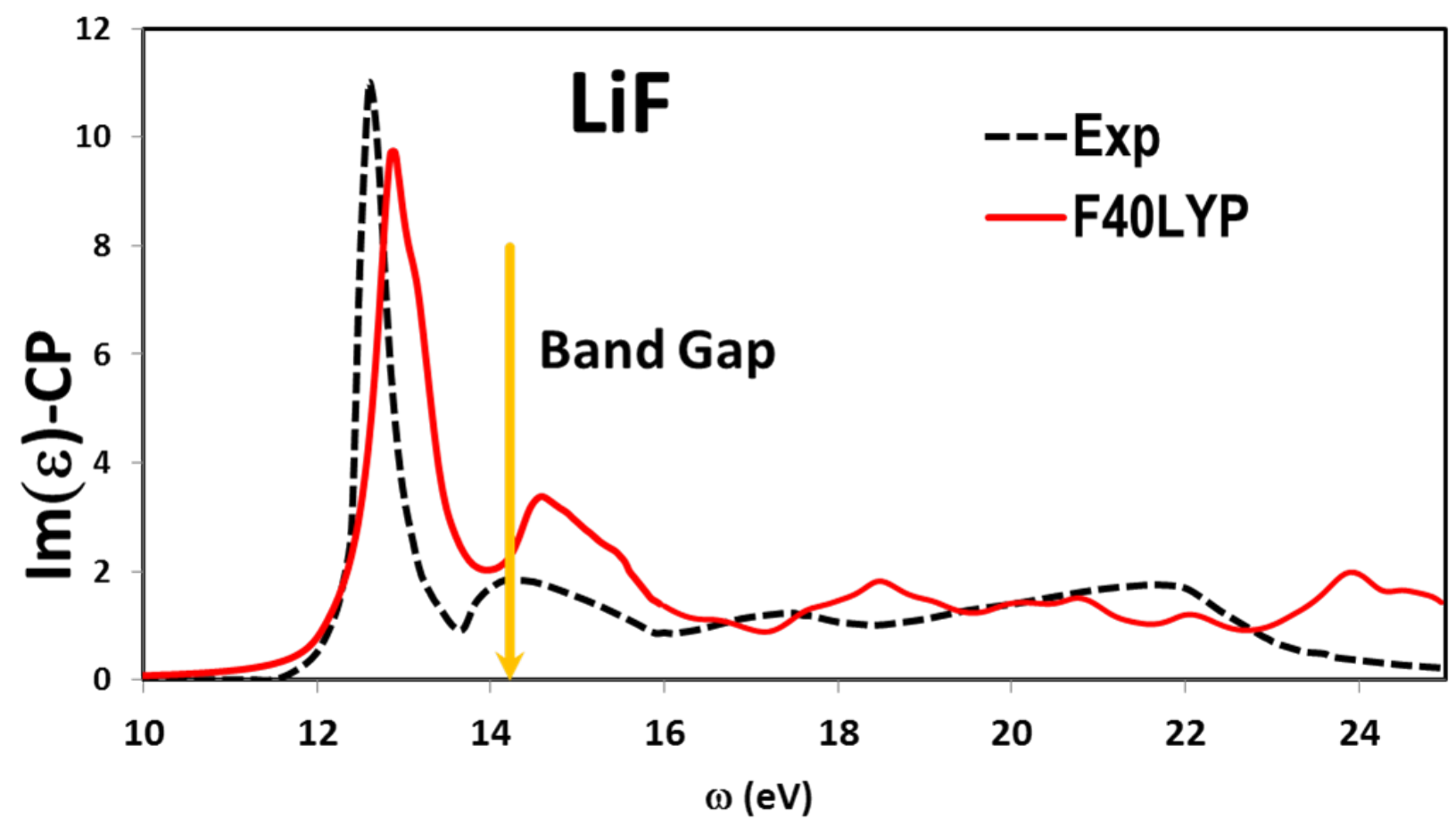

